Revista de Psicología y Educación / Journal of Psychology and Education, 2022, 17(1), 21-43 (www.rpye.es) Doi: https://doi.org/10.23923/rpye2022.01.214

ISSN: $1699-9517$

\title{
Instrumentos de Evaluación de Inteligencia Emocional en Educación Primaria: Una Revisión Sistemática
}

\author{
Rafael Sánchez-Camacho*y Mariona Grane
}

Universidad de Barcelona

\begin{abstract}
Resumen: Desde la aparición del término Inteligencia Emocional (IE), varios autores han desarrollado distintos modelos acompañados de cuestionarios de evaluación para medir el constructo. El objetivo de esta revisión sistemática fue analizar los instrumentos de evaluación de IE en el ámbito de la Educación Primaria (rango de edad 6-12 años) utilizados en investigaciones científicas publicadas en revistas indexadas. Se realizó una revisión sistemática para la síntesis de estudios siguiendo las pautas de PRISMA. La estrategia de búsqueda se llevó a cabo en cinco bases de datos: Dialnet, Psycinfo, Scopus, PyscNET y ERIC by ProQuest. Se encontraron un total de 62 artículos con 27 cuestionarios distintos para evaluar la IE en una población de 6 a 12 años. Los principales modelos teóricos de IE en los que se basaban los cuestionarios fueron: Modelo de Inteligencia Emocional-Social de Bar-On y Modelo de Salovey y Mayer. Los cuestionarios más utilizados fueron: The Bar-On Emotional Quotient Inventory (EQi:YV) y Trait Emotional Intelligence Questionnaire-Child Form (TEIQue-CF). Esta revisión sistemática recoge por primera vez los cuestionarios empleados específicamente para evaluar la IE de niños y los sintetiza en base a los diferentes modelos teóricos, contribuyendo al conocimiento de las herramientas disponibles para utilizar en el ámbito educativo.

Palabras clave: Inteligencia emocional, Educación primaria, Evaluación, Cuestionarios.
\end{abstract}

\section{Emotional Intelligence Assessments in Primary Education: A Systematic Review}

\begin{abstract}
Since the term Emotional Intelligence (EI) was coined, several authors have developed different theories accompanied by questionnaires to measure the construct. The objective of this systematic review was to analyze the El evaluation instruments in the field of Primary Education (age range 6-12 years) used in scientific research published in indexed journals. A systematic review was carried out following the PRISMA guidelines. The search strategy was carried out in five databases: Dialnet, Psycinfo, Scopus, PyscNET and ERIC by ProQuest. A total of 62 articles with 27 different questionnaires were found that evaluate $\mathrm{El}$ in a population with ages ranging from 6 to 12 years. The main theoretical models of $\mathrm{El}$ on which the questionnaires were based on are: the Bar-On model of emotional-social intelligence and the Salovey \& Mayer's model. The most widely used questionnaires were: The Bar-On Emotional Quotient Inventory (EQ-i: YV) and Trait Emotional Intelligence Questionnaire - Child Form (TEIQue - CF). This systematic review collects for the first time the questionnaires used specifically to evaluate children's $\mathrm{El}$ and synthesizes them based on the different theoretical models, contributing to the knowledge of the tools available for use in the educational field.

Key words: Emotional intelligence, Primary education, Evaluation, Questionnaires.
\end{abstract}

A pesar de que el término Inteligencia Emocional (IE) tiene varias definiciones dependiendo de los autores y sus modelos, todos parecen confluir en un origen similar:

Recibido: 03/10/2021 - Aceptado: 20/10/2021 - Avance online:30/12/2021 *Correspondencia: Rafael Sánchez Camacho

Universidad de Barcelona

Dirección: Campus de Mundet, Universitat de Barcelona, Passeig de la Vall d'Hebron, 171, 08035, Barcelona, España.

E-mail: rsanchca49@alumnes.ub.edu

Sánchez-Camacho, R. y Grané-Oro, M. (2022). Instrumentos de Evaluación de Inteligencia Emocional en Educación Primaria: Una Revisión Sistemática. Revista de Psicología y Educación, 17(1), 21-43, https://doi.org/10.23923/rpye2022.01.214 el concepto de inteligencia propuesto por Thorndike (1920). En su Modelo Multidimensional de Inteligencia, la Inteligencia Social es la capacidad que tienen las personas para entender, tratar y llevarse bien con la gente que les rodea. Es la parte de nuestra inteligencia que se utiliza para relacionarnos con efectividad. 
Posteriormente, Howard Gadner constituye la Teoría de las Inteligencias Múltiples en su libro "Frames of Mind" (1983). En esta teoría, postula la idea de que cada persona tiene siete tipos de inteligencias que le ayudan a relacionarse con su entorno. Entre los distintos tipos de inteligencias encontramos la Inteligencia Interpersonal (relaciones que establecen las personas entre sí) y la Inteligencia Intrapersonal (conocimiento de uno mismo y sus procesos). La unión de estos dos tipos de inteligencia da como resultado la IE que hoy en día conocemos.

El término IE aparece en la literatura por primera vez en el año 1990 de la mano de los psicólogos Salovey y Mayer. Según Salovey y Mayer (1997, p. 4) "la IE incluye la habilidad para percibir con precisión, valorar y expresar emoción; la habilidad de acceder y/o generar sentimientos cuando facilitan pensamientos; la habilidad de comprender la emoción y el conocimiento emocional; y la habilidad para regular las emociones para promover crecimiento emocional e intelectual". Sin embargo, es Daniel Goleman quien contribuyó a la difusión del concepto con la publicación de su libro Inteligencia Emocional (1995). Varios autores han ido desarrollando distintas teorías que han buscado definir el término (Bar-On, 1997, 2006; Bisquerra, 2007; Ekman, 1971; Furnham, 2003; Lane, 1987; Petrides, 2000). Dichas teorías utilizan diferentes cuestionarios o inventarios de evaluación para medir el constructo. La IE se comenzó a evaluar en adultos utilizando inicialmente el Trait MetaMood Scale (TMMS-48) (Salovey et al., 1995), y no fue hasta seis años después cuando se comenzaron a desarrollar cuestionarios para evaluar la IE de los niños. El primero, creado por Mayer et al. en el año 2005, se conoce por el nombre de Mayer-Salovey-Caruso Emotional Intelligence Test Young Version (MSCEIT-YV) (Mayer et al., 2005). A partir de ese momento, han surgido diferentes cuestionarios basados en distintas teorías para evaluar la IE en niños. Dependiendo del constructo en el que nos basemos o del instrumento que utilicemos, podemos hablar de instrumentos de medidas de IE como habilidad e instrumentos de medida como rasgo (también conocido como modelos mixtos). El primero hace referencia al uso de medidas objetivas para evaluar las capacidades óptimas de ejecución de una persona frente a tareas emocionales. Mientras que los cuestionarios de medida de rasgo se basan en autoinformes dónde se miden los atributos y percepciones subjetivas de los participantes (Agirre, 2017). Esta diferencia que nos ofrecen los distintos modelos ha sido motivo de controversia a la hora medir la IE a lo largo de los años. Los instrumentos que miden la IE como habilidad nos revelan si una persona tiene dichas habilidades desarrolladas o no, pero no nos indica si las aplica en su vida diaria. Sin embargo, los instrumentos de medida de rasgo o autoinformes sí muestran un grado de autoeficacia emocional (Pena y Repetto, 2008; Sánchez-Teruel y Robles-Bello, 2018). Existen revisiones actualizadas de instrumentos de IE que están principalmente focalizadas en población adulta $\left(\mathrm{O}^{\prime}\right.$ Connor et al. 2019) o en población general sin especificar por edades (Sánchez-Tervel y Robles-Bello, 2018); sin embargo, actualmente hay escaso conocimiento respecto a qué instrumentos se pueden utilizar para evaluar la IE en niños. El objetivo de esta revisión sistemática es analizar los instrumentos de evaluación de IE en el ámbito de la Educación Primaria (rango de edad 6-12 años) utilizados en investigaciones científicas publicadas en revistas indexadas. Este análisis tendrá en cuenta los modelos teóricos existentes detrás de cada cuestionario, las dimensiones que evalúa el cuestionario, el número de ítems y las edades en las que se han utilizado los cuestionarios.

\section{MÉTODO}

Realizamos una revisión sistemática integradora que permite la síntesis de estudios mixtos siguiendo los ítems de informes preferidos para revisiones sistemáticas y pautas de metaanálisis PRISMA (Eaton et al., 2017). Se llevó a cabo la estrategia de búsqueda en las bases de datos de Dialnet, Psycinfo, Scopus, PyscNET y ERIC by ProQuest hasta octubre de 2019 sin restricción del año de publicación. 
La estrategia de búsqueda se realizó en octubre de 2019 e incluyó las siguientes palabras clave: (emotional intelligence) AND child AND (questionnaire $O R$ tool $O R$ scale $O R$ measure) NOT (teenager OR adolescent $O R$ young adults). La estrategia de búsqueda se adaptó al lenguaje específico de cada base de datos.

\section{CRITERIO DE SELECCIÓN DE LOS ESTUDIOS}

Se incluyeron todos los artículos originales publicados en inglés o español que recogen una evaluación cuantitativa de la IE en niños, específicamente, de edades comprendidas entre los 6 y 12 años. Se recuperó un total de 1372 artículos de las búsquedas para revisión. Después de eliminar los duplicados (246), el proceso de selección del estudio fue realizado de forma independiente de acuerdo con las pautas de PRISMA (Eaton et al., 2017), siguiendo dos fases: revisión del título y abstract con Rayyan y revisión por texto completo. Para seleccionar los artículos se tuvieron en cuenta los siguientes criterios de inclusión y exclusión que aparecen en la Figura 1. Los criterios de inclusión fueron: artículos en los que se hayan utilizado cuestionarios de $\mathrm{IE}$, población de edades comprendidas entre los 6 y 12 años y artículos escritos en inglés y español. Los criterios de exclusión fueron: artículos no originales o no relacionados con el tema; artículos que evalúan a personas con discapacidad o desórdenes psicológicos y artículos que no evalúan la IE o evalúan la IE en adultos.

\section{PROCESO DE CODIFICACIÓN DE LOS ESTUDIOS}

El autor principal extrajo y sintetizó los datos de todos los estudios incluidos. El primer paso fue desarrollar una matriz para extraer las características principales de cada artículo: autor; año de publicación; país donde se realizó el estudio; muestra que

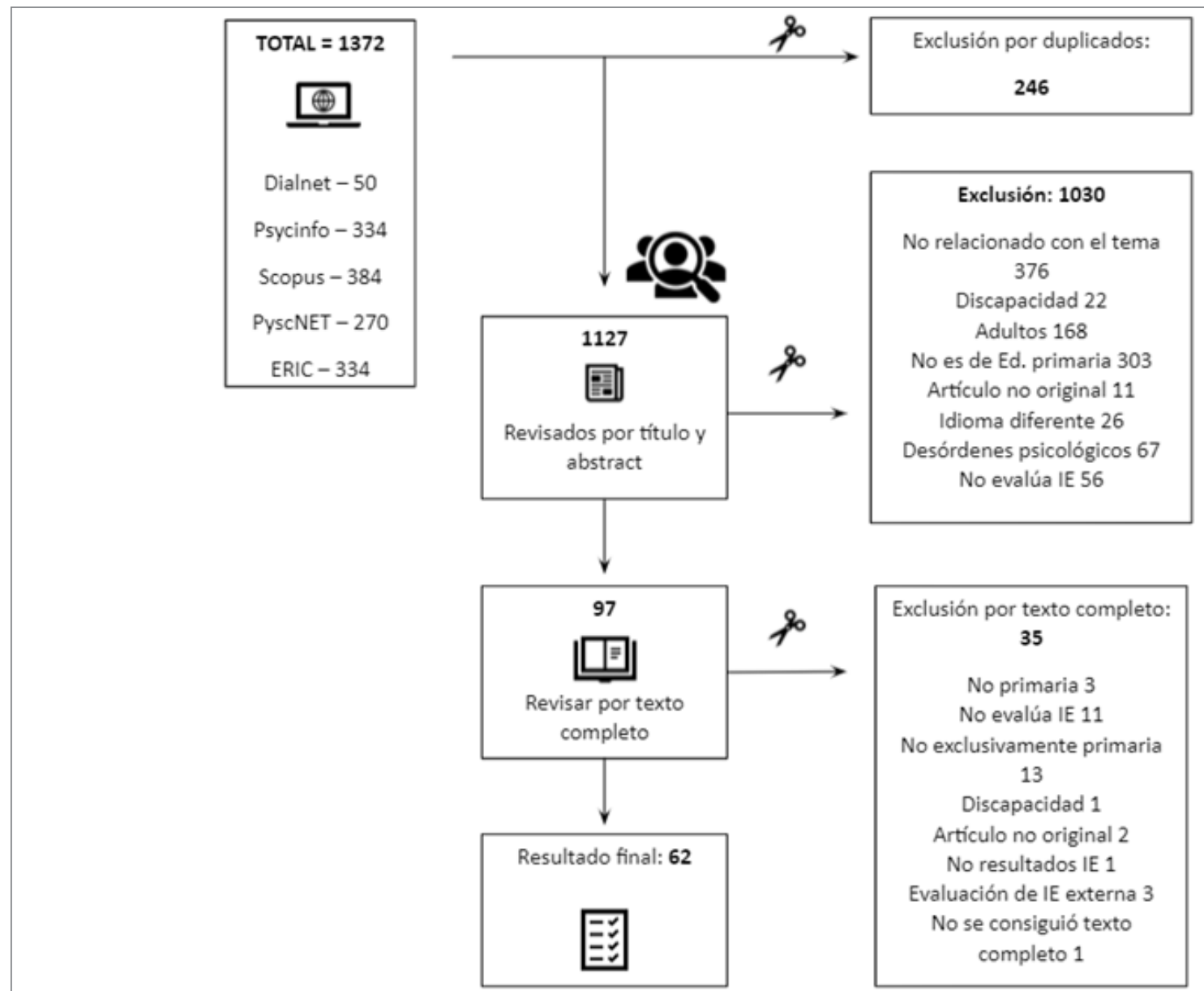

Figura 1: Diagrama de fujo de la selección de los artículos incluidos en la revisión. 
participó en el estudio (tamaño, edad y cursos escolares); método del estudio, instrumento para la evaluación de la IE (número de ítems, rango de evaluación), qué aspectos de la IE mide.

Se extrajeron los datos relacionados con los diferentes métodos de evaluación y luego se clasificó y sintetizó.

Tras la selección de los artículos, el resultado final fueron 62 artículos incluidos escritos en español o inglés. La mayoría de estos artículos fueron desarrollados en UK (18\%), Italia $(11,5 \%)$, España $(24,6 \%)$ y USA $(13,1 \%)$. El resto de artículos seleccionados pertenecían a los siguientes países: Australia, Bélgica, Corea, Egipto, Grecia, Holanda, India, Irán, Jordania, Líbano, Portugal, Serbia, Tailandia, Taiwán y Turquía. Los artículos incluidos fueron publicados entre los años 2005 y 2019.

\section{RESULTADOS}

Se encontraron un total de 27 cuestionarios para evaluar la IE en niños. Los nombres y las características principales de los cuestionarios encontrados se describen en la Tabla 1 de resultados. Los cuestionarios más utilizados fueron: The Bar-On Emotional Quotient Inventory (EQ-i:YV) (25,8\%), The Bar-On Emotional Quotient Inventory (BArOnICE:NA) (short version) (4,8\%), Trait Emotional Intelligence Questionnaire-Adolescent Short Form (TEIQue-ASF) (12,9\%), Trait Emotional Intelligence Questionnaire-Child Form (TEIQueCF) (22,6\%), Trait Emotional Intelligence Questionnaire-Child Short Form (TEIQue-CSF) (6,5\%), Mayer-Salovey-Caruso Emotional Intelligence Test - Youth Version (MSCEIT-YV) $(8,1 \%)$.

\section{Tabla 1}

Descripción de los instrumentos revisados para la medición de la inteligencia emocional en niños de 6 a 12 años

\begin{tabular}{|c|c|c|c|c|c|c|}
\hline $\begin{array}{c}\text { Cuestionario y } \\
\text { autor/es }\end{array}$ & $\begin{array}{l}\text { Modelo } \\
\text { teórico }\end{array}$ & Edad & Ítems & Dimensiones que mide & $\begin{array}{l}\text { Referencias (autor } \\
\text { artículo/Año) }\end{array}$ & $\begin{array}{l}\text { País en el } \\
\text { que se ha } \\
\text { utilizado }\end{array}$ \\
\hline $\begin{array}{l}\text { The Bar-On Emotional } \\
\text { Quotient Inventory } \\
\text { (EQ-i:M) } \\
\text { Bar-On y Parker, (2000) }\end{array}$ & $\begin{array}{c}\text { Modelo de } \\
\text { inteligencia } \\
\text { emocional-social } \\
\text { de Bar-On (1997; } \\
\text { 2006) }\end{array}$ & $\begin{array}{l}+ \text { de } 7 \\
\text { años }\end{array}$ & 60 & $\begin{array}{l}5 \text { subescalas y puntuación total: } \\
\text { Escala intrapersonal } \\
\text { Escala interpersonal } \\
\text { Escala de manejo del estrés } \\
\text { Escala de adaptabilidad } \\
\text { Estado de ánimo }\end{array}$ & $\begin{array}{c}\text { El Hassan et al. (2005) } \\
\text { Tannous et al. (2010) } \\
\text { Asl Aminabadi et al. (2011; 2013) } \\
\text { Ferrándiz et al. (2012) } \\
\text { Alegre (2012a; } 2012 \mathrm{~b}) \\
\text { Brouzos et al. (2014) } \\
\text { dos Santos et al. (2014) } \\
\text { Parisi et al. (2017) } \\
\text { Bordás et al. (2019) } \\
\text { Operto et al. (2019) } \\
\text { Windingstad et al. (2011) } \\
\text { Sánchez et al. (2014) } \\
\text { De las Heras et al. (2017) } \\
\text { Esturgó-Deu et al. (2019) }\end{array}$ & $\begin{array}{l}\text { Líbano } \\
\text { Jordania } \\
\text { Irŕn } \\
\text { España } \\
\text { Estados Unidos } \\
\text { Grecia } \\
\text { Portugal } \\
\text { Itallia }\end{array}$ \\
\hline $\begin{array}{l}\text { The Bar-On Emotional } \\
\text { Quotient Inventory } \\
\text { (BArOn-ICE:NA) (short } \\
\text { version) } \\
\text { Ugariza y Pajares, } \\
\text { (2004) }\end{array}$ & $\begin{array}{c}\text { Modelo de } \\
\text { inteligencia } \\
\text { emocional-social } \\
\text { de Bar-On (1997; } \\
\text { 2006) }\end{array}$ & $\begin{array}{l}+ \text { de } 7 \\
\text { años }\end{array}$ & 30 & $\begin{array}{l}5 \text { subescalas y puntuación total: } \\
\text { Escala intrapersonal } \\
\text { Escala interpersonal } \\
\text { Escala de manejo del estrés } \\
\text { Escala de adaptabilidad }\end{array}$ & $\begin{array}{l}\text { Pérez-Escoda et al. (2012) } \\
\text { Carillo et al. (2018) } \\
\text { El Hassan et al. (2014) }\end{array}$ & $\begin{array}{l}\text { España- } \\
\text { Estados Unidos } \\
\text { España } \\
\text { Líbano }\end{array}$ \\
\hline $\begin{array}{l}\text { Test of Emotion } \\
\text { Comprehension (TEC) } \\
\text { Pons et al., (2004) }\end{array}$ & Panteórica & $\begin{array}{c}+ \text { de } 3 \\
\text { años }\end{array}$ & 17 & $\begin{array}{c}9 \text { componentes: } \\
\text { Reconocimiento de las emocional } \\
\text { Comprensión de las causas externas de las } \\
\text { emociones } \\
\text { Comprensión del deseo despertado por } \\
\text { la emoción } \\
\text { Comprensión de las emociones en función } \\
\text { de las creencias } \\
\text { Comprensión de la influencia del } \\
\text { recuerdo en la evaluación de los estados } \\
\text { emocionales } \\
\text { Comprensión de la posibilildad de controlar } \\
\text { las experiencias emocionales } \\
\text { Comprensión de la posibilidad de } \\
\text { esconder un estado emocional } \\
\text { Comprensión de la existencia de } \\
\text { respuestas emocionales múltiples o hasta } \\
\text { contradictorias (ambivivalentes) para una } \\
\text { situación } \\
\text { Comprensión de expresiones morales. } \\
\text { Moralidad) }\end{array}$ & dos Santos et al. (2014) & Portugal \\
\hline
\end{tabular}


Tabla 1/Continuación)

Descripción de los instrumentos revisados para la medición de la inteligencia emocional en niños de 6 a 12 años

\begin{tabular}{|c|c|c|c|c|c|c|}
\hline $\begin{array}{c}\text { Cuestionario y } \\
\text { autor/es }\end{array}$ & $\begin{array}{l}\text { Modelo } \\
\text { teórico }\end{array}$ & Edad & Ítems & Dimensiones que mide & $\begin{array}{l}\text { Referencias (autor } \\
\text { artículo/Año) }\end{array}$ & $\begin{array}{l}\text { País en el } \\
\text { que se ha } \\
\text { utilizado }\end{array}$ \\
\hline $\begin{array}{l}\text { Trait Emotional } \\
\text { Intelligence } \\
\text { Questionnaire- } \\
\text { Adolescent Short Form } \\
\text { (TEIQue-ASF) } \\
\text { Petrides et al., } \\
\text { (2006) }\end{array}$ & $\begin{array}{l}\text { Modelo de } \\
\text { Autoeficiencia } \\
\text { Emocional } \\
\text { de Petridesy } \\
\text { Furnham, 2000; } \\
\text { 2003 }\end{array}$ & $\begin{array}{l}+ \text { de } 11 \\
\text { años }\end{array}$ & 30 & $\begin{array}{c}15 \text { subescalas: } \\
\text { Adaptabilidad } \\
\text { Asertividad } \\
\text { Percepción de la emoción (uno mismo } \\
\text { y otros) } \\
\text { Expresión de la emoción } \\
\text { Manejo de la emoción (Otros) } \\
\text { Regulación de la emoción } \\
\text { Impulsividad } \\
\text { Relaciones } \\
\text { Autoestima } \\
\text { Automotivación } \\
\text { Conciencia social } \\
\text { Manejo del estrés } \\
\text { Empatía del rasgo } \\
\text { Felicidad del rasgo } \\
\text { Rasgo optimismo }\end{array}$ & $\begin{array}{l}\text { Petrides et al. (2006) } \\
\text { Williams et al. (2009; 2010a; 2010b) } \\
\text { Jellesma et al. (2011) } \\
\text { Ferrando et al. (2011) } \\
\text { Ferrándiz et al. (2012) } \\
\text { Kokkinos. (2011) }\end{array}$ & $\begin{array}{l}\text { Reino Unido } \\
\text { Países Bajos } \\
\text { España } \\
\text { Grecia }\end{array}$ \\
\hline $\begin{array}{l}\text { Trait Emotional } \\
\text { Intelligence } \\
\text { Questionnaire-Child } \\
\text { Form (TEIQue-CF) } \\
\text { Mavroveli et al., (2009) }\end{array}$ & $\begin{array}{l}\text { Modelo de } \\
\text { Autoeficiencia } \\
\text { Emocional } \\
\text { de Petridesy } \\
\text { Furnham, 2000; } \\
\quad 2003\end{array}$ & $\begin{array}{l}8-12 \\
\text { años }\end{array}$ & $83-75$ & $\begin{array}{c}\text { 9 subescalas: } \\
\text { Adaptabilidad } \\
\text { Disposición afectiva } \\
\text { Expresión emocional } \\
\text { Percepción de la emoción } \\
\text { Regulación de las emociones } \\
\text { Baja impulsividad } \\
\text { Relaciones entre pares } \\
\text { Autoestima } \\
\text { Automotivación }\end{array}$ & $\begin{array}{l}\text { Mavroveli et al. (2008; 2009; 2011) } \\
\text { Qualter et al. (2011) } \\
\text { Agnoli et al. (2012; 2019) } \\
\text { Hansenne (2012) } \\
\text { Russo et al. (2012) } \\
\text { Banjac et al. (2016) } \\
\text { Abo Elellla et al. (2017) } \\
\text { Pandya. (2017) } \\
\text { Li et al. (2017) } \\
\text { Davis et al. (2019) } \\
\text { Mancini (2019) }\end{array}$ & $\begin{array}{l}\text { Reino Unido } \\
\text { Italia } \\
\text { Bélgica } \\
\text { Serbia } \\
\text { Egipto } \\
\text { India } \\
\text { Estados Unidos } \\
\text { Itallia }\end{array}$ \\
\hline $\begin{array}{l}\text { Trait Emotional } \\
\text { Intelligence } \\
\text { Questionnaire-Child } \\
\text { Short Form (TEIQue-CSF) } \\
\text { Mavroveli et al., (2009) }\end{array}$ & $\begin{array}{l}\text { Modelo de } \\
\text { Autoeficiencia } \\
\text { Emocional } \\
\text { de Petrides y } \\
\text { Furnham, 2000; } \\
\quad 2003\end{array}$ & $\begin{array}{l}8-12 \\
\text { años }\end{array}$ & 36 & $\begin{array}{l}\text { Adaptabililad } \\
\text { Disposición afectiva } \\
\text { Expresión emocional } \\
\text { Percepción de la emoción } \\
\text { Regulación de las emociones } \\
\text { Bajaimpulsividad } \\
\text { Relaciones entre pares } \\
\text { Autoestima } \\
\text { Automotivación }\end{array}$ & $\begin{array}{l}\text { Babalis et al. (2013) } \\
\text { Peachey et al. (2017) } \\
\text { Stassart et al. (2017a; 2017b) }\end{array}$ & $\begin{array}{l}\text { Grecia } \\
\text { Estados Unidos } \\
\text { Bélgica }\end{array}$ \\
\hline $\begin{array}{l}\text { Trait Emotional } \\
\text { Intelligence } \\
\text { Questionnaire-Child } \\
\text { Form } \\
\text { (TElQue-CF) (adapted } \\
\text { on Digital Based-Game) } \\
\text { Chi Yang et al., (2019) }\end{array}$ & $\begin{array}{l}\text { Modelo de } \\
\text { Autoeficiencia } \\
\text { Emocional } \\
\text { de Petrides y } \\
\text { Furnham, 2000; } \\
\quad 2003\end{array}$ & $\begin{array}{l}9-10 \\
\text { años }\end{array}$ & 9 & Rasgo de IE & Yang et al. (2019) & Taiwán \\
\hline $\begin{array}{l}\text { The Schutte Self-Report } \\
\text { Emotional Intelligence } \\
\text { (SSREI) } \\
\text { Schutte et al., (1998) }\end{array}$ & $\begin{array}{l}\text { Modelo de } \\
\text { Habilidades } \\
\text { de Inteligencia } \\
\text { Emocional de } \\
\text { Salovey y Mayer } \\
\text { (1990) }\end{array}$ & $\begin{array}{l}\text { No } \\
\text { validado } \\
\text { para } \\
\text { niños }\end{array}$ & 33 & $\begin{array}{l}\text { Percepción emocional. } \\
\text { Facilitación emocional del pensamiento. } \\
\text { Comprensión emocional. } \\
\text { Regulación emocional. }\end{array}$ & Williams et al. $\left(2009 ; 2010^{9}\right)$ & Reino Unido \\
\hline $\begin{array}{l}\text { Mayer-Salovey-Caruso } \\
\text { Emotional Intelligence } \\
\text { Test - Youth Version } \\
\text { (MSCEIT-W) } \\
\text { Mayer, Salovey y } \\
\text { Caruso, (2005) }\end{array}$ & $\begin{array}{l}\text { Modelo de } \\
\text { Habilidades } \\
\text { de Inteligencia } \\
\text { Emocional de } \\
\text { Salovey y Mayer } \\
\text { (1990; 1997) }\end{array}$ & $\begin{array}{l}+ \text { de } 8 \\
\text { años }\end{array}$ & $\begin{array}{l}184- \\
101\end{array}$ & $\begin{array}{l}\text { Percepción emocional. } \\
\text { Facilitación emocional del pensamiento. } \\
\text { Comprensión emocional. } \\
\text { Regulación emocional. }\end{array}$ & $\begin{array}{l}\text { Windingstad et al. (2011) } \\
\text { Duncan et al. (2013) } \\
\text { Davis et al. (2019) } \\
\text { Qualter et al. (2011; 2019) }\end{array}$ & $\begin{array}{l}\text { Estados Unidos } \\
\text { Reino Unido }\end{array}$ \\
\hline $\begin{array}{l}\text { Trait- Meta-Mood } \\
\text { Scale-24 (TMMS-24) } \\
\text { Fernández-Berrocal, et } \\
\text { al., (2004) basado en } \\
\text { Salovey et al., (1995) }\end{array}$ & $\begin{array}{l}\text { Modelo de } \\
\text { Habilidades } \\
\text { de Inteligencia } \\
\text { Emocional de } \\
\text { Salovey y Mayer } \\
\text { (1990; 1997) }\end{array}$ & $\begin{array}{l}\text { No } \\
\text { validado } \\
\text { para } \\
\text { niños }\end{array}$ & 24 & $\begin{array}{l}3 \text { subescalas: } \\
\text { Atención emocional } \\
\text { Claridad de sentimientos } \\
\text { Reparación emocional }\end{array}$ & Ferragut et al. (2012) & España \\
\hline
\end{tabular}




\section{Tabla 1/Continuación)}

Descripción de los instrumentos revisados para la medición de la inteligencia emocional en niños de 6 a 12 años

\begin{tabular}{|c|c|c|c|c|c|c|}
\hline $\begin{array}{c}\text { Cuestionario y } \\
\text { autor/es }\end{array}$ & $\begin{array}{l}\text { Modelo } \\
\text { teórico }\end{array}$ & Edad & Ítems & Dimensiones que mide & $\begin{array}{c}\text { Referencias (autor } \\
\text { artículo/Año) }\end{array}$ & $\begin{array}{l}\text { País en el } \\
\text { que se ha } \\
\text { utilizado }\end{array}$ \\
\hline $\begin{array}{l}\text { Trait- Meta-Mood Scale } \\
\text { for Children (TMMSC) } \\
\text { Rockhill y Greener, } \\
\text { (1999) }\end{array}$ & $\begin{array}{l}\text { Modelo de } \\
\text { Habilidades } \\
\text { de Inteligencia } \\
\text { Emocional de } \\
\text { Salovey y Mayer } \\
\text { (1990; 1997) }\end{array}$ & $\begin{array}{l}\text { De } 8 \text { a } \\
12 \text { años }\end{array}$ & 16 & $\begin{array}{l}3 \text { subescalas: } \\
\text { Atención emocional } \\
\text { Claridad de sentimientos } \\
\text { Reparación emocional }\end{array}$ & Alegre (2012a; 2012b) & Estados Unidos \\
\hline $\begin{array}{l}\text { The Emotion } \\
\text { Recognition and } \\
\text { Perception test (ERP) } \\
\text { Stylianou, (2007) }\end{array}$ & $\begin{array}{l}\text { Modelo de } \\
\text { Reconocimiento } \\
\text { de Emociones } \\
\text { de Ekman y } \\
\text { Friesen, } 1971\end{array}$ & $\begin{array}{l}\text { No } \\
\text { validado } \\
\text { para } \\
\text { niños }\end{array}$ & 24 fotos & $\begin{array}{l}\text { etiquetado afectivo (reconocimiento) } \\
\text { toma de perspectiva afectiva (percepción) }\end{array}$ & Qualter et al. (201 1) & Reino Unido \\
\hline $\begin{array}{l}\text { Brief Emotional } \\
\text { Intelligence Scale } \\
\text { (BEIS-10) } \\
\text { Davies et al., (2010) }\end{array}$ & $\begin{array}{c}\text { Modelo de } \\
\text { Habilidades } \\
\text { de Inteligencia } \\
\text { Emocional de } \\
\text { Salovey y Mayer } \\
\text { (1990) }\end{array}$ & $\begin{array}{l}\text { No } \\
\text { validado } \\
\text { para } \\
\text { niños }\end{array}$ & 10 & $\begin{array}{l}5 \text { subescalas } \\
\text { Evaluación de las propias emociones } \\
\text { Evaluación de las emociones de los demás } \\
\text { Regulación de las propias emociones } \\
\text { Regulación de las emociones de los } \\
\text { demás } \\
\text { Uso de emociones }\end{array}$ & $\begin{array}{l}\text { Hsieh et al. (2019) } \\
\text { Huang et al. (2017) }\end{array}$ & $\begin{array}{l}\text { Taiwán } \\
\text { Taiwán }\end{array}$ \\
\hline $\begin{array}{l}\text { The Prevalence of Using } \\
\text { Emotional Intelligence } \\
\text { in Students Scale } \\
\text { Titrek, (2005) }\end{array}$ & $\begin{array}{l}\text { Modelo de } \\
\text { Inteligencia } \\
\text { Emocional de } \\
\text { Goleman (1995) }\end{array}$ & - & 35 & $\begin{array}{c}\text { Conciencia } \\
\text { Manejo de emociones } \\
\text { Emociones motivadoras } \\
\text { Empatía } \\
\text { Habilidades sociales }\end{array}$ & Akca (2010) & Turquía \\
\hline $\begin{array}{l}\text { Student Character } \\
\text { Survey (SCS) } \\
\text { Rutgers University }\end{array}$ & Panteórica & - & 18 & $\begin{array}{c}\text { Concepto de Inteligencia Emocional } \\
\text { global }\end{array}$ & van Dyke et al. (2008) & Estados Unidos \\
\hline $\begin{array}{l}\text { The evaluation of } \\
\text { emotional perception } \\
\text { and emotional } \\
\text { comprehension in } \\
\text { children } \\
\text { EMOCINE Test (had-oc) } \\
\text { Sastre et al., (2019) }\end{array}$ & $\begin{array}{l}\text { Modelo de } \\
\text { Habilidades } \\
\text { de Inteligencia } \\
\text { Emocional de } \\
\text { Salovey y Mayer } \\
\text { (1990; 1997) }\end{array}$ & $\begin{array}{l}\text { De } 8-13 \\
\text { años }\end{array}$ & 15 & $\begin{array}{l}\text { Percepción emocional } \\
\text { Comprensión emocional }\end{array}$ & Sastre et al. (2019) & España \\
\hline $\begin{array}{c}\text { Emotion } \\
\text { Awareness } \\
\text { Questionnaire (EAQ30) } \\
\text { Rieffe et al., (2007; } \\
\text { 2008) }\end{array}$ & Panteórica & $\begin{array}{l}+ \text { de } 9 \\
\text { años }\end{array}$ & 30 & $\begin{array}{c}6 \text { subescalas: } \\
\text { Emociones diferenciadoras } \\
\text { Conciencia corporal } \\
\text { Comunicación de emociones } \\
\text { Expresión contundente (Acting Out } \\
\text { Emotions); } \\
\text { Atención y análisis a las emociones de } \\
\text { los demás } \\
\text { Atención y análisis de las emociones } \\
\text { propias }\end{array}$ & $\begin{array}{l}\text { Stassart et al. (2017a) } \\
\text { Ordóñez et al. (2015) }\end{array}$ & $\begin{array}{l}\text { Bélgica } \\
\text { España }\end{array}$ \\
\hline $\begin{array}{l}\text { Levels of Emotional } \\
\text { Awareness Scale for } \\
\text { Children (LEAS-C) } \\
\text { Bajgar et al., (2005) }\end{array}$ & $\begin{array}{l}\text { Modelo de } \\
\text { Niveles de } \\
\text { Conciencia } \\
\text { Emocional de } \\
\text { Lane y Schwartz } \\
\text { (1987) }\end{array}$ & $\begin{array}{l}+ \text { de } 8 \\
\text { años }\end{array}$ & 12 & $\begin{array}{c}3 \text { subescalas: } \\
\text { Conciencia emocional propia } \\
\text { Conciencia emocional de los demás. } \\
\text { Conciencia emocional total }\end{array}$ & Agnoli et al. (2019) & Italia \\
\hline
\end{tabular}


Tabla 7/Continuación)

Descripción de los instrumentos revisados para la medición de la inteligencia emocional en niños de 6 a 12 años

\begin{tabular}{|c|c|c|c|c|c|c|}
\hline $\begin{array}{c}\text { Cuestionario y } \\
\text { autor/es }\end{array}$ & $\begin{array}{l}\text { Modelo } \\
\text { teórico }\end{array}$ & Edad & Ítems & Dimensiones que mide & $\begin{array}{c}\text { Referencias (autor } \\
\text { artículo/Año) }\end{array}$ & $\begin{array}{l}\text { País en el } \\
\text { que se ha } \\
\text { utilizado }\end{array}$ \\
\hline $\begin{array}{c}\text { Test } \\
\text { de Inteligencia Social } \\
\text { Emocional } \\
\text { Chiriboga y Franco, } \\
\text { (2001) }\end{array}$ & $\begin{array}{l}\text { Modelo de } \\
\text { Inteligencia } \\
\text { Emocional de } \\
\text { Goleman } 1996\end{array}$ & 10 años & 60 & $\begin{array}{c}5 \text { subescalas: } \\
\text { Autoconciencia } \\
\text { Autocontrol } \\
\text { Automotivación o aprovechamiento } \\
\text { emocional } \\
\text { Empatía } \\
\text { Habilidad social }\end{array}$ & Mateu-Martínez et al. (2013) & España \\
\hline $\begin{array}{l}\text { Cuestionario elaborado } \\
\text { para evaluar la IE } \\
\text { como conjunto de } \\
\text { habilidades (ad-hoc) } \\
\text { Pulido-Acosta y Herrera- } \\
\text { Clavero, (2016) }\end{array}$ & Panteórica & $\begin{array}{l}+ \text { de } 6 \\
\text { años }\end{array}$ & 65 & $\begin{array}{l}5 \text { subescalas: } \\
\text { Conocimiento de sí mismo } \\
\text { Autoconcepto } \\
\text { Motivación } \\
\text { Empatía } \\
\text { Autocontrol emocional }\end{array}$ & $\begin{array}{l}\text { Pulido-Acosta y Herrera-Clavero; } \\
\qquad(2019)\end{array}$ & España \\
\hline $\begin{array}{l}\text { The Emotional Skills } \\
\text { and Competence } \\
\text { Questionnaire (ESCQ) } \\
\text { Takšić, (2002; 2009) }\end{array}$ & $\begin{array}{l}\text { Modelo de } \\
\text { Habilidades } \\
\text { de Inteligencia } \\
\text { Emocional de } \\
\text { Mayer, Caruso, } \\
\text { y Salovey } 2016\end{array}$ & $\begin{array}{l}\text { No } \\
\text { validado } \\
\text { para } \\
\text { niños }\end{array}$ & $\begin{array}{l}45 \\
\text { items }\end{array}$ & $\begin{array}{l}3 \text { subescalas: } \\
\text { Percepción y comprensión emocional } \\
\text { Expresión y etiquetado emocional } \\
\text { Control y gestión emocional }\end{array}$ & Turanzas et al. (2020) & España \\
\hline $\begin{array}{l}\text { Emotional Intelligence } \\
\text { Scale (EIS) } \\
\text { Moon, (1996; 1997) }\end{array}$ & $\begin{array}{l}\text { Modelo de } \\
\text { Habilidades } \\
\text { de Inteligencia } \\
\text { Emocional de } \\
\text { Salovey y Mayer, } \\
1990\end{array}$ & $\begin{array}{l}+ \text { de } 8 \\
\text { años }\end{array}$ & $\begin{array}{l}47 \\
\text { items }\end{array}$ & $\begin{array}{l}\text { 4 subescalas: } \\
\text { Percibir emoción } \\
\text { Comprender la emoción } \\
\text { Razonar con emoción } \\
\text { Manejar la emoción }\end{array}$ & Kim et al. (2017) & Corea \\
\hline Moon, (1996; 1997) & $\begin{array}{c}\text { Inteligencia } \\
\text { Emocional de } \\
\text { Salovey y Mayer, } \\
1990\end{array}$ & & & $\begin{array}{l}\text { Razonar con emoción } \\
\text { Manejar la emoción }\end{array}$ & & \\
\hline $\begin{array}{l}\text { Desarrollo de } \\
\text { Inteligencia Emocional } \\
\text { (ad-hoc) } \\
\text { D'Amico, (2018) }\end{array}$ & $\begin{array}{c}\text { Modelo de } \\
\text { Habilidades } \\
\text { de Inteligencia } \\
\text { Emocional de } \\
\text { Salovey y Mayer } \\
\text { (1997) }\end{array}$ & $\begin{array}{l}\text { De } 8 \text { a } \\
12 \text { años }\end{array}$ & $\begin{array}{l}27 \\
\text { items }\end{array}$ & $\begin{array}{l}\text { 4 subescalas: } \\
\text { Percibir emoción } \\
\text { Comprender la emoción } \\
\text { Razonar con emoción } \\
\text { Manejar la emoción }\end{array}$ & D’Amico; (2018) & Italia \\
\hline $\begin{array}{l}\text { Cuestionario de } \\
\text { Desarrollo Emocional } \\
\text { López y Pérez, (2010) }\end{array}$ & $\begin{array}{c}\text { Modelo de } \\
\text { Competencia } \\
\text { Emocional de } \\
\text { Bisquerra, } 2000 \\
\text { y } 2007\end{array}$ & $\begin{array}{l}\text { De } 10 \text { a } \\
12 \text { años }\end{array}$ & $\begin{array}{c}38 \\
\text { items }\end{array}$ & $\begin{array}{c}5 \text { subescalas: } \\
\text { conciencia emocional } \\
\text { Regulación emocional } \\
\text { Autonomía emocional } \\
\text { Competencia social } \\
\text { Competencias para la vida y el bienestar }\end{array}$ & Ros et al. (2017) & España \\
\hline $\begin{array}{l}\text { Emotional Competence } \\
\text { Observation Instrument } \\
\text { (Instrumento had-oc) } \\
\text { Pérez-Escoda, (2012) }\end{array}$ & $\begin{array}{c}\text { Modelo de } \\
\text { Competencia } \\
\text { Emocional de } \\
\text { Bisquerra y Pérez } \\
\text { (2007) }\end{array}$ & $\begin{array}{l}+ \text { de } 6 \\
\text { años }\end{array}$ & 79 & $\begin{array}{c}5 \text { subescalas: } \\
\text { Conciencia Emocional. } \\
\text { Regulación Emocional. } \\
\text { Autonomía Emocional. } \\
\text { Competencia Social. } \\
\text { Competencias para la vida y el bienestar. }\end{array}$ & Pérez-Escoda et al. (2012) & $\begin{array}{l}\text { España-Estados } \\
\text { Unidos }\end{array}$ \\
\hline $\begin{array}{l}\text { The Sullivan Emotional } \\
\text { Intelligence Scale for } \\
\text { Children (SEISC) } \\
\text { Sullivan, (1999) }\end{array}$ & $\begin{array}{c}\text { Modelo de } \\
\text { Habilidades } \\
\text { de Inteligencia } \\
\text { Emocional de } \\
\text { Salovey y Mayer } \\
\text { (1997) }\end{array}$ & - & $\begin{array}{c}22 \\
\text { items }\end{array}$ & $\begin{array}{l}\text { Reconocer la emoción } \\
\text { Entender la emoción } \\
\text { Manejar la emoción }\end{array}$ & Pandya (2017) & India \\
\hline $\begin{array}{l}\text { Thai Emotional } \\
\text { Intelligence Screening } \\
\text { Test (TEIST) } \\
\text { Thai Department of } \\
\text { Mental Health (2000) } \\
\text { Sucaromana, (2010) }\end{array}$ & Panteórica & $\begin{array}{c}+ \text { de } 12 \\
\text { años }\end{array}$ & $\begin{array}{c}52 \\
\text { items }\end{array}$ & $\begin{array}{c}\text { Subescalas: } \\
\begin{array}{c}\text { Virtud (autocontrol emocional, empatía y } \\
\text { responsabilidad) }\end{array} \\
\text { Felicidad (Paz, satisfacción de vida y } \\
\text { autoestima). } \\
\text { Competencia (Automotivación, relación } \\
\text { interpersonal y resolución de conflictos) }\end{array}$ & laosanurak et al. (2016) & Tailandia \\
\hline
\end{tabular}


Tabla 2

Número de ítems de cada uno de los cuestionarios de Inteligencia Emocional revisados.

\begin{tabular}{|c|c|c|c|c|}
\hline \multirow{2}{*}{ Cuestionario } & \multicolumn{4}{|c|}{ Rango de ítems } \\
\hline & $0-30$ & $31-60$ & $61-100$ & + de 100 \\
\hline Bar-On Emotional Quotient Inventory (EQ-i:Y) & & $x$ & & \\
\hline Bar-On Emotional Quotient Inventory (BarOn-ICE:NA) (short version) & $x$ & & & \\
\hline Emotional Competence Observation Instrument (had-oc) & & & $x$ & \\
\hline Trait Emotional Intelligence Questionnaire_-Adolescent Short Form (TEIQue-ASF) & $x$ & & & \\
\hline Trait Emotional Intelligence Questionnaire-Child Form (TEIQue-CF) & & & $x$ & \\
\hline Trait Emotional Intelligence Questionnaire-Child Short Form (TEIQue-CSF) & & $x$ & & \\
\hline \multicolumn{5}{|l|}{ Trait Emotional Intelligence Questionnaire-Child Form } \\
\hline (TEIQue-CF) (adapted on Digital Based-Game) & $x$ & & & \\
\hline Mayer-Salovey-Caruso Emotional Intelligence Test - Youth Version (MSCEIT-YV) & & & & $x$ \\
\hline Trait- Meta-Mood Scale-24 (TMMS-24) & $x$ & & & \\
\hline Trait- Meta-Mood Scale for Children (TMMSC) & $x$ & & & \\
\hline The Emotion Recognition and Perception test (ERP) & $x$ & & & \\
\hline \multicolumn{5}{|l|}{ The evaluation of emotional perception and emotional } \\
\hline comprehension in children EMOCINE Test (had-oc) & $x$ & & & \\
\hline Emotion Awareness Questionnaire (EAQ30) & $x$ & & & \\
\hline Levels of Emotional Awareness Scale for Children (LEAS-C) & $x$ & & & \\
\hline Test de Inteligencia Social Emocional & & $x$ & & \\
\hline Cuestionario elaborado para evaluar la IE como conjunto de habilidades (ad-hoc) & & & $x$ & \\
\hline Emotional Intelligence Scale (EIS) by Moon & & $x$ & & \\
\hline Desarrollo de Inteligencia Emocional (ad-hoc) & $x$ & & & \\
\hline Cuestionario de Desarrollo Emocional & & $x$ & & \\
\hline Thai Emotional Intelligence Screening Test (TEIST) & & $x$ & & \\
\hline Test of Emotion Comprehension (TEC) & $x$ & & & \\
\hline The Sullivan Emotional Intelligence Scale for Children (SEISC) & $x$ & & & \\
\hline The Schutte Self-Report Emotional Intelligence (SSREI) & & $x$ & & \\
\hline Brief Emotional Intelligence Scale (BEIS-10) & $x$ & & & \\
\hline The Prevalence of Using Emotional Intelligence in Students Scale. & & $x$ & & \\
\hline Student Character Survey (SCS) & $x$ & & & \\
\hline The Emotional Skills and Competence Questionnaire (ESCQ) & & $x$ & & \\
\hline Porcentaje total & $51,85 \%$ & $33,33 \%$ & $11,11 \%$ & $3,7 \%$ \\
\hline
\end{tabular}


Los cuestionarios presentaban un rango de ítems que abarca desde los 9 a 184 ítems. El $51,85 \%$ de cuestionarios contaba con menos 30 ítems, el 33,33\% contenía entre 31 a 60 ítems, el $11,11 \%$ de 61 a 100 ítems y el 3,7\% más de 100 ítems. En la Tabla 2, se muestran el rango de ítems para cada uno de los cuestionarios incluidos en la revisión.

Los principales modelos teóricos de IE en los que se basaban los cuestionarios encontrados fueron: Modelo de Salovey y Mayer (1990; 1997), Modelo de Mayer, Caruso, y Salovey (2016), Modelo de Petrides y Furnham, (2000; 2003), Modelo de Bar-On (1997; 2006), Modelo de Bisquerra y Pérez (2007), Modelo de Ekman y Friesen (1971), Modelo de Goleman (1995; 1996) y Modelo de Lane y Schwartz (1987). También se encontraron algunos cuestionarios basados en una visión panteórica (aplicación de varias teorías para el desarrollo de un instrumento). En la Tabla 3 se muestran los porcentajes y el número de estudios que han utilizado cada uno de los modelos.

\section{CUESTIONARIOS BASADOS EN EL MODELODE HABILIDADES DE INTELIGENCIA EMOCIONAL DE SALOVEY Y MAYER}

Este modelo ha sido el más utilizado a la hora de desarrollar cuestionarios. En total 10 cuestionarios $(37 \%)$ de los encontrados se basaron en este modelo, que originalmente incluye 4 dimensiones: percepción emocional, facilitación emocional del pensamiento, comprensión emocional y regulación emocional. Con el cuestionario MSCEIT-YV (Salovey y Mayer, 2005) se pueden evaluar dichas dimensiones.

Los otros 9 cuestionarios que se pueden utilizar para evaluar este modelo son: Brief Emotional Intelligence Scale (BEIS-10) ( Davies et al., 2010); Trait- Meta-Mood Scale-24 (TMMS-24) (Fernández-Berrocal et al., 2004); Trait- Meta-Mood Scale for Children (TMMSC) (Rockhill y Greener, 1999); The Schutte Self-Report Emotional Intelligence (SSREI) (Schutte et al., 1998); The Sullivan Emotional Intelligence Scale for Children (SEISC)

Tabla 3

Número de estudios y porcentajes en los que se emplea cada modelo teórico.

\begin{tabular}{|c|c|c|}
\hline Modelo teórico & $\begin{array}{c}\text { Porcentaje de los estudios en los que } \\
\text { se utiliza }\end{array}$ & $\begin{array}{c}\text { Número de estudios que han } \\
\text { utilizado este modelo }\end{array}$ \\
\hline Modelo de Inteligencia Emocional-Social & 30,64 & 27 \\
\hline Modelo de Autoeficiencia Emocional & 43,55 & 17 \\
\hline Modelo de Habilidades de Inteligencia & 27,42 & 2 \\
\hline Emocional & 1,61 & 2 \\
\hline Modelo de Reconocimiento de & 3,22 & 1 \\
\hline Modelo de Inteligencia Emocional & 3,22 & \\
\hline Modelo de Competencia Emocional & 1,61 & 6 \\
\hline Emocional & 9,68 & \\
\hline
\end{tabular}


(Sullivan, 1999); The evaluation of emotional perception and emotional comprehension in children EMOCINE Test (ad-hoc) (Sastre et al., 2019); The Emotional Skills and Competence Questionnaire (ESCQ) (Takšić, 2002; 2009); Emotional Intelligence Scale (EIS) (Moon, 1996; 1997) y el cuestionario de desarrollo de inteligencia emocional (adhoc) creado por D'Amico (2018). El número de dimensiones que se evalúan en estos cuestionarios es muy heterogéneo. El número mínimo de dimensiones evaluadas fueron dos: percepción emocional y comprensión emocional, evaluadas por el EMOCINE Test (Sastre et al., 2019) y cómo máximo se evaluaron cinco dimensiones: evaluación de las propias emociones, evaluación de las emociones de los demás, regulación de las propias emociones, regulación de las emociones de los demás y uso de emociones, evaluadas por el BEIS-10 (Davies et al., 2010) (Tabla 1).

\section{CUESTIONARIOS BASADOS EN EL MODELO DE INTELIGENCIA EMOCIONAL-SOCIAL DE BAR-ON}

Este modelo se puede evaluar por medio de los cuestionarios EQ-i:YV y Bar-On-ICE:NA. El EQ-i:YV consta de 60 ítems mientras que el Bar-On-ICE:NA es una versión corta que consta de 30 ítems. Ambos cuestionarios miden cinco subescalas del modelo de IE del autor (escala intrapersonal, interpersonal, manejo del estrés, adaptabilidad y estado de ánimo) y, la suma de estas cinco subescalas da una puntuación total en IE, también llamado Cociente Emocional (CE).

\section{CUESTIONARIOS BASADOS EN EL MODELO DE AUTOEFICIENCIA EMOCIONAL DE PETRIDES Y FURNHAM}

Este modelo puede ser medido por 4 cuestionarios desarrollados por los autores. EI TEIQue-ASF creado en 2006 consta de 30 ítems de respuesta que mide las 15 subescalas del modelo: adaptabilidad, asertividad, percepción de la emoción (uno mismo y otros), expresión de la emoción, manejo de la emoción (otros), regulación de la emoción, impulsividad, relaciones, autoestima, automotivación, conciencia social, manejo del estrés, empatía del rasgo, felicidad del rasgo y rasgo optimismo. Este cuestionario está dirigido a una población de 11 años en adelante.

El TEIQue-CF desarrollado por Mavroveli et al. en 2008, consta de 83 ítems, aunque algunos autores han utilizado este cuestionario pasando solo 75 ítems. En él se miden 9 subescalas del modelo: adaptabilidad, disposición afectiva, expresión emocional, percepción de la emoción, regulación de las emociones, baja impulsividad, relaciones entre pares, autoestima y automotivación. Este cuestionario tuvo una versión corta llamada TEIQue-CSF que consta de 36 ítems para medir las mismas 9 subescalas. Está destinado a una población de 8 a 12 años. Otra de las adaptaciones que tuvo este cuestionario fue la realizada por Yang et al. en 2019. Este cuestionario se modificó para adaptarse al contexto del estudio. En él se redujeron el número de ítems a 9 que medían la IE total de los participantes. Este cuestionario fue utilizado con niños de 9-10 años, pero no ha sido validado en esta población.

\section{CUESTIONARIOS BASADOS EN EL MODELO DE COMPETENCIA EMOCIONAL DE BISQUERRA Y PÉREZ}

El Modelo de Competencia Emocional de Bisquerra y Pérez (2007) es medido a través de los cuestionarios Emotional Competence Observation Instrument y el Cuestionario de Desarrollo Emocional. Ambos cuestionarios miden las cinco subescalas del modelo de los autores: conciencia emocional, regulación emocional. autonomía emocional, competencia social y competencias para la vida y el bienestar. El primero de ellos está orientado a medir la IE de niños de más de 6 años y consta de un total de 79 ítems. El segundo, está orientado a medir las competencias emocionales en niños de 10 a 12 años y consta de un total de 38 ítems. 


\section{CUESTIONARIOS BASADOS EN EL MODELO DE INTELIGENCIA EMOCIONAL DE GOLEMAN}

El Modelo de la Inteligencia Emocional de Goleman (1995) ha sido medida utilizando 2 cuestionarios. The Prevalence of Using Emotional Intelligence in Students Scale (Titrek, 2005) fue utilizado en niños de edades comprendidas entre los 7-14 años, mientras que el cuestionario Test de Inteligencia Social Emocional (Chiriboga y Franco, 2001) está dirigido a una población de mayores de 10 años. Ambos cuestionarios miden las 5 subescalas del modelo: autoconciencia, manejo de emociones, motivación de emociones, empatía y habilidades sociales.

\section{CUESTIONARIOS BASADOS EN EL MODELO DE RECONOCIMIENTO DE EMOCIONES DE EKMAN Y FRIESEN}

El Modelo de Reconocimiento de Expresiones Faciales de las emociones definidas por Ekman (1971) fue utilizado por Qualter et al. (2011) para evaluar el reconocimiento y la percepción emocional en niños de edades de 8-11 años. Para evaluar estas dimensiones se utilizaron dos tipos de pruebas. La primera fue mediante preguntas abiertas en las que se les pedía a los niños el reconocimiento de las emociones básicas en las expresiones faciales de 24 fotografías (Stylianou, 2007). La segunda prueba fue The Emotional Recognition Vignettes (Camras et al., 1988), que consta de 30 viñetas en las que se les pide a los participantes que decidan qué palabra o sentimiento describe mejor la escena. Estas escenas cubren las seis categorías emocionales definidas por Ekman (sorpresa, miedo, tristeza, alegría, rabia y asco).

\section{CUESTIONARIO BASADO EN EL MODELO DE NIVELES DE CONCIENCIA EMOCIONAL DE LANE Y SCHWARTZ}

El modelo teórico de Niveles de Conciencia Emocional (LEA) que propusieron Lane y Schwartz (1987) sostiene que las experiencias emocionales se vuelven más diferenciadas e integradas con la edad, con representaciones de estados emocionales pasando de formas implícitas y explícitas (Agnoli, 2019). Este modelo puede medirse con el cuestionario Levels of Emotional Awareness Scale for Children (LEAS-C) que consta de 12 escenarios representativos de la vida cotidiana entre dos personas. En cada escenario se describen de dos a cuatro oraciones que involucran a dos personas. Los escenarios se organizan en torno a cuatro emociones (ira, miedo, felicidad y tristeza). Los participantes tienen que describir los propios sentimientos y los de la otra persona para cada escenario. Cada escenario tiene asignado tres puntuaciones, que reflejan 3 subescalas: conciencia emocional propia, conciencia emocional de los demás y conciencia emocional total.

\section{CUESTIONARIOS SIGUIENDO UNA APROXIMACIÓN PANTEÓRICA}

El 18,5\% de los cuestionarios encontrados en esta revisión estaban basados en una aproximación panteórica (Tabla 1). En estos estudios se hablaba de varías teorías y modelos de IE pero no se especificaba cómo se había llegado a definir las dimensiones del constructo de cada cuestionario. Estos cuestionarios son: Thai Emotional Intelligence Screening Test (TEIST) (Sucaromana, 2010); Cuestionario para evaluar la IE como conjunto de habilidades (ad-hoc) de (Pulido-Acosta y Herrera-Clavero, 2019); Emotion Awareness Questionnaire (EAQ30) (Rieffe et al., 2007; 2008); Student Character Survey (SCS) (Van Dyke, 2008) y Test of Emotion Comprehension (TEC) (Pons et al., 2004).

\section{USO DE LOS CUESTIONARIOS EN EL CONTEXTO DE LA EDUCACIÓN PRIMARIA}

En la Tabla 4 se muestran las edades y etapas educativas en las que han sido validados y utilizados cada uno de los cuestionarios.

Los cuestionarios que se muestran en la Tabla 5, a pesar de haber sido utilizados en una población infantil, no han sido validados para su uso. 
Tabla 4

Cuestionarios y cursos escolares en los que ha sido utilizado.

\begin{tabular}{|c|c|c|c|c|c|c|c|}
\hline \multirow{3}{*}{ Cuestionarios } & \multicolumn{7}{|c|}{ Cursos escolares } \\
\hline & Pre escolar & $\begin{array}{l}\text { Primer } \\
\text { curso }\end{array}$ & $\begin{array}{l}\text { Segundo } \\
\text { curso }\end{array}$ & $\begin{array}{l}\text { Tercer } \\
\text { curso }\end{array}$ & $\begin{array}{l}\text { Cuarto } \\
\text { curso }\end{array}$ & $\begin{array}{l}\text { Quinto } \\
\text { curso }\end{array}$ & $\begin{array}{l}\text { Sexto } \\
\text { curso }\end{array}$ \\
\hline & $\begin{array}{l}\text { De } 3 \text { a } 6 \\
\text { años }\end{array}$ & $\begin{array}{l}\text { 6-7 } \\
\text { años }\end{array}$ & 7-8 años & 8-9 años & 9-10 años & $\begin{array}{l}\text { 10-11 } \\
\text { años }\end{array}$ & $\begin{array}{l}11-12 \\
\text { años }\end{array}$ \\
\hline $\begin{array}{l}\text { Bar-On Emotional Quotient } \\
\text { Inventory (EQ-i:Y) }\end{array}$ & & & $x$ & $x$ & $x$ & $x$ & $x$ \\
\hline $\begin{array}{l}\text { Bar-On Emotional Quotient } \\
\text { Inventory (BArOn-ICE:NA) (short } \\
\text { version) }\end{array}$ & & & $x$ & $x$ & $x$ & $x$ & $x$ \\
\hline $\begin{array}{l}\text { Emotional Competence } \\
\text { Observation Instrument (had- } \\
\text { oc) }\end{array}$ & & $x$ & $x$ & $x$ & $x$ & $x$ & $x$ \\
\hline $\begin{array}{l}\text { Trait Emotional Intelligence } \\
\text { Questionnaire_Adolescent } \\
\text { Short Form (TEIQue-ASF) }\end{array}$ & & & & & & $x$ & $x$ \\
\hline $\begin{array}{l}\text { Trait Emotional Intelligence } \\
\text { Questionnaire-Child Form } \\
\text { (TElQue-CF) }\end{array}$ & & & & $x$ & $x$ & $x$ & $x$ \\
\hline $\begin{array}{l}\text { Trait Emotional Intelligence } \\
\text { Questionnaire-Child Short } \\
\text { Form (TEIQue-CSF) }\end{array}$ & & & & $x$ & $x$ & $\mathrm{x}$ & $x$ \\
\hline $\begin{array}{l}\text { Trait Emotional Intelligence } \\
\text { Questionnaire-Child Form }\end{array}$ & & & & & & & \\
\hline $\begin{array}{l}\text { (TEIQue-CF) (adapted on } \\
\text { Digital Based-Game) }\end{array}$ & & & & & $x$ & & \\
\hline $\begin{array}{l}\text { Mayer-Salovey-Caruso } \\
\text { Emotional Intelligence Test - } \\
\text { Youth Version (MSCEIT-Y) }\end{array}$ & & & & $x$ & $x$ & $\mathrm{x}$ & $x$ \\
\hline $\begin{array}{l}\text { Trait- Meta-Mood Scale for } \\
\text { Children (TMMSC) }\end{array}$ & & & & $x$ & $x$ & $x$ & $x$ \\
\hline $\begin{array}{c}\text { Recognize emotions (facial } \\
\text { expressions) }\end{array}$ & & & & $x$ & $x$ & $x$ & \\
\hline $\begin{array}{l}\text { Student Character Survey } \\
\text { (SCS) }\end{array}$ & & & & & & $x$ & $x$ \\
\hline $\begin{array}{l}\text { The evaluation of emotional } \\
\text { perception and emotional } \\
\text { comprehension in children } \\
\text { EMOCINE Test (had-oc) }\end{array}$ & & & & $x$ & $x$ & $x$ & $x$ \\
\hline
\end{tabular}


Tabla 4 (Continuación)

Cuestionarios y cursos escolares en los que ha sido utilizado.

\begin{tabular}{|c|c|c|c|c|c|c|c|}
\hline \multirow{3}{*}{ Cuestionarios } & \multicolumn{7}{|c|}{ Cursos escolares } \\
\hline & Pre escolar & $\begin{array}{l}\text { Primer } \\
\text { curso }\end{array}$ & $\begin{array}{l}\text { Segundo } \\
\text { curso }\end{array}$ & $\begin{array}{l}\text { Tercer } \\
\text { curso }\end{array}$ & $\begin{array}{l}\text { Cuarto } \\
\text { curso }\end{array}$ & $\begin{array}{l}\text { Quinto } \\
\text { curso }\end{array}$ & $\begin{array}{l}\text { Sexto } \\
\text { curso }\end{array}$ \\
\hline & $\begin{array}{l}\text { De } 3 \text { a } 6 \\
\text { años }\end{array}$ & $\begin{array}{l}\text { 6-7 } \\
\text { años }\end{array}$ & 7-8 años & 8-9 años & 9-10 años & $\begin{array}{l}10-11 \\
\text { años }\end{array}$ & $\begin{array}{l}11-12 \\
\text { años }\end{array}$ \\
\hline $\begin{array}{l}\text { Emotion Awareness } \\
\text { Questionnaire (EAQ30) }\end{array}$ & & & & & $x$ & $x$ & $x$ \\
\hline $\begin{array}{l}\text { Levels of Emotional Awareness } \\
\text { Scale for Children (LEAS-C) }\end{array}$ & & & & $\mathrm{x}$ & $\mathrm{x}$ & $x$ & $\mathrm{x}$ \\
\hline $\begin{array}{l}\text { Test de Inteligencia Social } \\
\text { Emocional }\end{array}$ & & & & & & $x$ & \\
\hline $\begin{array}{l}\text { Cuestionario elaborado para } \\
\text { evaluar la IE como conjunto } \\
\text { de habilidades (ad-hoc) }\end{array}$ & & $x$ & $x$ & $x$ & $x$ & $x$ & $x$ \\
\hline $\begin{array}{l}\text { Emotional Intelligence Scale } \\
\text { (EIS) by Moon }\end{array}$ & & & & $\mathrm{x}$ & $x$ & $x$ & $x$ \\
\hline $\begin{array}{l}\text { Desarrollo de Inteligencia } \\
\text { Emocional (ad-hoc) }\end{array}$ & & & & $x$ & $x$ & $x$ & $x$ \\
\hline $\begin{array}{c}\text { Cuestionario de Desarrollo } \\
\text { Emocional }\end{array}$ & & & & & & $x$ & $x$ \\
\hline $\begin{array}{l}\text { Thai Emotional Intelligence } \\
\text { Screening Test (TEIST) }\end{array}$ & & & & & & & $x$ \\
\hline $\begin{array}{c}\text { Test of Emotion } \\
\text { Comprehension (TEC) }\end{array}$ & $x$ & $x$ & $x$ & $x$ & $x$ & $x$ & $x$ \\
\hline $\begin{array}{l}\text { The Sullivan Emotional } \\
\text { Intelligence Scale for Children } \\
\text { (SEISC) }\end{array}$ & & & & $x$ & $x$ & $x$ & $x$ \\
\hline
\end{tabular}


Tabla 5

Edades en las que han sido utilizados los cuestionarios no validados

\begin{tabular}{|c|c|}
\hline Cuestionario & Edades en las que se ha utilizado \\
\hline The Prevalence of Using Emotional Intelligence in Students Scale & Población de 7-14 años \\
\hline Brief Emotional Intelligence Scale (BEIS-10) & Población 9-10 años \\
\hline The Emotional Skills and Competence Questionnaire (ESCQ) & Población de 8 a 14 años \\
\hline Trait- Meta-Mood Scale-24 (TMMS-24) & Población de 9-12 años \\
\hline The Schutte Self-Report Emotional Intelligence (SSREI) & Población de 10-11 años \\
\hline
\end{tabular}

\section{DISCUSIÓN}

El objetivo de esta revisión sistemática fue analizar los instrumentos de evaluación de IE en el ámbito de la Educación Primaria (rango de edad 6-12 años) utilizados en investigaciones científicas publicadas en revistas indexadas. En esta revisión sistemática se encontraron un total de 27 cuestionarios que han sido utilizados para evaluar la IE en niños de educación primaria.

No todos los cuestionarios de IE encontrados están traducidos al español. De los 27 cuestionarios encontrados en esta revisión sistemática, solo 11 han sido utilizados en España. Los cuestionarios de IE para niños más utilizado en España han sido EQ-i:YV (Bar-On y Parker, 2000), Bar-On-ICE:NA (Ugarriza y Pajares, 2004) y TEIQue-ASF (Petrides et al., 2006). Otros cuestionarios como TEIQue-CF (Mavroveli et al., 2009) y TEIQue-CSF (Mavroveli et al., 2009) o MSCEIT-YV (Mayer et al., 2005) aún no han sido traducidos al español, por lo que en futuros estudios sería recomendable poder validar este tipo de cuestionarios en nuestro contexto cultural para disponer de una mayor cantidad de cuestionarios para utilizar y valorar dimensiones complementarias, aspectos culturales y modelos teóricos distintos de la IE en niños.
Cuando hablamos del contexto infantil, es importante realizar estudios que demuestren la validez de los cuestionarios en esta población específica. Algunos estudios incluidos en esta revisión sistemática han utilizado cuestionarios creados y validados en población adulta para evaluar la IE en una población de edades comprendidas entre los 7 y 14 años. No es recomendable hacer uso de este tipo de cuestionarios ya que existen cuestionarios específicos diseñados y/o adaptados a población infantil. Por ejemplo, en el estudio realizado por Ferragut et al. en 2012 se utilizó la versión del TMMS-24 (FernándezBerrocal, et al., 2004) para adultos cuando existen versiones específicas para niños como el TMMSC (Rockhill y Greener, 1999). Esto podría ser un indicio de que en algunos casos no se utilizan cuestionarios específicos y adaptados para cada tipo de población.

Al utilizar un cuestionario con población infantil también es importante tener en cuenta la longitud o número de ítems que presenta el cuestionario. El 48,14\% de los cuestionarios encontrados en esta revisión sistemática contenían más de 30 ítems. Algunos de los cuestionarios de IE más extensos disponen de versiones breves. El cuestionario Bar-OnICE:NA (Ugarriza y Pajares, 2004) en su versión corta consta de 30 ítems, la mitad de ítems que la versión normal, siendo igualmente válido 
para evaluar las dimensiones de IE del modelo de Bar-On. Otra opción de cuestionario en su versión corta es el Trait Emotional Intelligence Questionnaire (Mavroveli et al., 2009), tanto en su versión de adolescentes como para niños. La versión corta para adolescentes consta de un total de 30 ítems que mide 15 subescalas del modelo de IE de Petrides y Furnham, mientras que la versión para niños consta de un total de 36 ítems que mide 9 subescalas del mismo modelo de IE. Además, en esta revisión sistemática se ha encontrado que existen adaptaciones no validadas de los cuestionarios originales que han sido utilizadas para evaluar rasgos específicos. Por ejemplo, en el caso de la adaptación digital gamificada del TEIQue-CF (Yang et al., 2019), el cuestionario se vio reducido a un total de 9 ítems que se utilizaron para medir el rasgo de IE de los participantes. A pesar de no ser un cuestionario validado, en futuras investigaciones sería interesante validar nuevas adaptaciones de cuestionarios con menos ítems y que utilicen otras vías más atractivas y sencillas para la población infantil como la gamificación de cuestionarios.

El primer paso para poder adaptar un cuestionario es comprender el modelo teórico en el que se basa. Los modelos de IE más utilizados para el desarrollo de los cuestionarios encontrados en esta revisión sistemática fueron el Modelo de Habilidades de Inteligencia Emocional de Salovey y Mayer, el Modelo de Inteligencia Emocional-Social de Bar-On y el Modelo de Autoeficiencia Emocional de Petrides y Furnham. Esto a su vez coincide con los cuestionarios más utilizados en los distintos estudios, que han sido: EQ-i:YV (Bar-On y Parker, 2000), TEIQue-CF (Mavroveli et al., 2009), TEIQueASF (Petrides et al., 2006) y MSCEIT-YV (Mayer et al., 2005). Los cuestionarios y pruebas de IE basados en los modelos de habilidades revelan en sus resultados si los participantes tienen desarrolladas o no estas habilidades o competencias, pero no revelan en sus resultados si son aplicadas en su vida diaria (Pena y Repeto, 2008). El ejemplo más significativo de cuestionario para medir la IE como habilidad son los cuestionarios TMMS24 (Fernández-Berrocal, et al., 2004) - TMMSC (Rockhill y Greener, 1999), basados en el modelo de Salovey y Mayer. Sin embargo, si lo que queremos evaluar o conocer es el grado de autoeficiencia emocional, utilizaremos cuestionarios basados en modelos mixtos o de rasgo como son el cuestionario Bar-OnICE:NA (Ugarriza y Pajares, 2004) o TEIQueCF (Mavroveli et al., 2009). Esta diferencia que ofrecen los cuestionarios frente a las pruebas de habilidad podría suponer una cierta ventaja si queremos conocer cómo un niño utiliza sus habilidades emocionales en situaciones de su día a día, y a su vez, ayudar a desarrollar programas de educación emocional más eficaces (Sánchez-Teruel, 2009).

La proliferación de diferentes modelos, tanto de habilidad como de rasgo, y sus correspondientes formas de evaluar el constructo, han dificultado su aplicación en el ámbito educativo. A pesar de que diversos estudios plantean la relación de la IE de niños relacionados con el rendimiento académico (Guil et al. 2011 ) y con el bienestar (Extremera et al. 2011; Salguero et al., 2011), no se ha planteado ningún marco común para la creación de un constructo que permita introducir el concepto en el currículo escolar.

En el marco estratégico para la cooperación europea en el ámbito de la educación y la formación (Consejo de la Unión Europea, 2021) no aparece reflejada la importancia de desarrollar o trabajar competencias relacionadas con la IE basada en cualquiera de los modelos expuestos en esta revisión sistemática, a pesar del creciente interés por el desarrollo de la IE en los últimos veinte años (Ciarrochi et al., 2006; Prieto et al., 2008), especialmente en edades tempranas (Barchard, 2003). En el sistema educativo español, sí se hace referencia a la importancia del desarrollo emocional y afectivo de los alumnos desde edades tempranas, aunque no hace referencia específica de cómo trabajarlos (LOMLOE, 2020). Esta revisión puede ayudar a aumentar el conocimiento 
de las herramientas disponibles para evaluar la IE y así permitir a la comunidad educativa desarrollar programas educativos que permitan a los alumnos trabajar la IE y poder cuantificar los resultados de sus programas.

Esta revisión sistemática recoge por primera vez los cuestionarios empleados específicamente para evaluar la IE de niños de edades comprendidas entre los 6 y 12 años y los sintetiza en base a los diferentes modelos teóricos. Entre las limitaciones encontradas en esta revisión, se encuentra la posible omisión de otros modelos de IE que pudieran no aparecer en las bases de datos utilizadas o el hecho de que haya cuestionarios que no utilicen el término IE como tal y que, por lo tanto, no apareciesen en la búsqueda realizada.

Se puede concluir que en general, ha crecido el interés por la implementación de la IE en las aulas a pesar de las limitaciones existentes (Aguaded et al., 2017; Extremera et al., 2019; Contreras, 2021; Costa et al., 2021). Sin embargo, aún quedan muchos pasos por recorrer hasta su definitiva implementación en el currículo. Para poder implementar satisfactoriamente programas que trabajen la IE en las escuelas desde edades tempranas, es fundamental consensuar una definición y una teoría o modelo, así como establecer cuáles son las habilidades o competencias que se tienen que desarrollar y su correspondiente instrumento de evaluación. Además, no se puede aplicar eficazmente un programa sin la participación de la comunidad educativa, especialmente docentes, y con una formación adecuada.

\section{- Conflicto de intereses}

Los autores declaran no tener ningún conflicto de interés.

\section{- Financiación}

La presente investigación no ha recibido ayudas específicas provenientes de agencias del sector público, sector comercial o entidades sin ánimo de lucro.

\section{REFERENCIAS}

* Referencia de publicación incluida en la muestra de publicaciones revisadas.

* Abo Elella, E., Hassan, G. A., Sabry, W., Hendawy, H., Shorub, E., Zyada, F., y Medany, O. (2017). Trait emotional intelligence in a sample of Egyptian children with attention deficit hyperactivity disorder. Child and Adolescent Mental Health, 22(4), 216-223. DOl: https://doi. org/10.1111/camh.12236

Agirre, I. A., Etxaniz, I. E., y Fernández, A. R. (2017). La medida de la inteligencia emocional en el ámbito psicoeducativo. Revista interuniversitaria de formación del profesorado, (88), 53-64.

* Agnoli, S., Mancini, G., Andrei, F., y Trombini, E. (2019). The relationship between trait emotional intelligence, cognition, and emotional awareness: An interpretative model. Frontiers in psychology, 10. DOI: https://doi. org/10.3389/fpsyg.2019.01711

* Agnoli, S., Mancini, G., Pozzoli, T., Baldaro, B., Russo, P. M., y Surcinelli, P. (2012). The interaction between emotional intelligence and cognitive ability in predicting scholastic performance in school-aged children. Personality and Individual Differences, 53(5), 660-665. DOI: https://doi. $\mathrm{org} / 10.1016 /$ i.paid.2012.05.020

Aguaded Gómez, M. C., y Valencia, J. (2017). Estrategias para potenciar la inteligencia emocional en educación infantil: aplicación del modelo de Mayer y Salovey. Tendencias pedagógicas. https:// doi.org/10.15366/tp2017.30.010

* Akca, F. (2010). Talented and average intelligent children's levels of using emotional intelligence. Procedia-Social and Behavioral Sciences, 5, 553558. DOI: https://doi.org/10.1016/i. sbspro.2010.07.141

* Aminabadi, N. A., Adhami, Z. E., Oskovei, S. G., Najafpour, E., y Jamali, Z. (2013). Emotional intelligence subscales: Are they correlated with child anxiety and behavior in the dental setting?. Journal of Clinical Pediatric Dentistry, 38(1), 61 66. DOI: https://doi.org/10.17796/ jcpd.38.1.k754h164m3210764

* Alegre, A. (2012a). Is there a relation between mothers' parenting styles and childrens' trait emotional intelligence?

* Alegre, A. (2012b, October). The relation between the time mothers and children spent together and the children's trait 
emotional intelligence. In Child \& Youth Care Forum (Vol. 41, No. 5, pp. 493. 508). Springer US. DOI: https://doi. org/10.1007/s10566-012-9180-z

* Asl Aminabadi, N., Erfanparast, L., Ebrahim Adhami, Z., Maliaii, E., Ranjbar, F., y Jamali, Z. (2011). The impact of emotional intelligence and intelligence quotient (IQ) on child anxiety and behavior in the dental setting. Acta Odontologica Scandinavica, 69(5), 292-298. DOI: https://doi.org/10. $3109 / 00016357.2011 .568959$

* Babalis, T., Tsoli, K., Artikis, C. T., MylonakouKeke, I., y Xanthakou, Y. (2013). The Impact of Social and Emotional Learning Programs on the Emotional Competence and Academic Achievement of Children in Greek Primary School. World Journal of Education, 3(6), 54-63.

Baigar, J., Ciarrochi, J., Lane, R. D., y Deane, F. (2005). Development of the levels of emotional awareness scale for children (LEAS-C). Br. J. Dev. Psychol. 23, 569-586. DOl: https://doi. org/10.1348/026151005X35417

* Banjac, S., Hull, L., Petrides, K. V., y Mavroveli, S. (2016). Validation of the Serbian adaptation of the trait emotional intelligence questionnaire-child form (TEIQue-CF). psihologiia, 49(4), 375392.

Barchard, K. A. (2003). Does emotional intelligence assist in the prediction of academic success?. Educational and psychological measurement, 63(5), 840-858. DOl: https://doi. org/10.1177/0013164403251333

Bar-On, R. (1997). Development of the BarOn EQ-i: a measure of emotional and social intelligence. Comunicación presentada en la 105 Convención Anual de la Asociación Americana de Psicología. Chicago, Illinois. Estados Unidos.

Bar-On, R. (1997). The emotional quotient inventory (EQ-I): A test emotional intelligence. Toronto: Multi-Health Systems.

Bar-On, R. (2006). The Bar-On model of emotional-social intelligence (ESI). Psicothema, 18(supl), 13-25.

Bisquerra R., y Escoda, N. P. (2007). Las competencias emocionales. Educación $X X 1,(10), 61-82$.
* Bordás, C. S., Rábanos, N. L., y Supervía, P. U. (2019). Inteligencia emocional y creatividad en alumnos de $1^{\circ}$ y $2^{\circ}$ de Primaria. $\dot{2}$ Existen diferencias por género?. Contextos Educativos. Revista de Educación, (24), 181-196. DOl: https://doi.org/10.18172/con.3530

* Brouzos, A., Misailidi, P., y Hadjimattheou, A. (2014). Associations between emotional intelligence, socio-emotional adjustment, and academic achievement in childhood: The influence of age. Canadian Journal of School Psychology, 29(2), 83-99. DOI: https://doi. org/10.1177/0829573514521976

Camras, L. A., Ribordy, S., Hill, J., Martino, S., Spaccarelli, S., y Stefani, R. (1988). Recognition and posing of emotional expressions by abused children and their mothers. Developmental Psychology, 24(6), 776. DOl: https://doi. $\mathrm{org} / 10.1037 / 0012-1649.24 .6 .776$

* Carrillo, A., Estévez, C., y GómezMedina, M. D. (2018). ¿̇Influyen las prácticas educativas en el desarrollo de la inteligencia emocional de sus hijos? Revista INFAD de Psicología. International Journal of Developmental and Educational Psychology., $1(1), 203$ 212. DOI: https://doi.org/10.17060/ ijodaep.2018.n1.v1.1190

Chiriboga, R. D., y Franco, J. E. (2001). Validación de un test de Inteligencia Emocional en niños de diez años de edad. Revista Médico de Familia, 9, 24 38.

Resolución del Consejo relativa a un marco estratégico para la cooperación europea en el ámbito de la educación y la formación con miras al Espacio Europeo de Educación y más allá (2021-2030). DOUE-Z-2021-70017.26 de febrero de 2021.

Contreras-Lima, J. J. (2021). La inteligencia emocional para el desarrollo de aprendizaje en los niños de nivel inicial. [Tesis de Carrera de Ciencias de la Educación, Universidad Técnica de Machala]. http://repositorio.utmachala.edu.ec/ bitstream/48000/16855/1/E-12231 CONTRERAS\%20LIMA\%20JESSICA $\% \overline{2} 0$ JACQUELINE\%281\%29.pdf 
Costa Rodriguez, C., Palma Leal, X., y Salgado Farías, C. (2021). Docentes emocionalmente inteligentes. Importancia de la Inteligencia Emocional para la aplicación de la Educación Emocional en la práctica pedagógica de aula. Estudios pedagógicos (Valdivia), 47(1), 219-233. DOI: http://dx.doi.org/10.4067/s0718$\underline{07052021000100219}$

* D'Amico, A. (2018). The Use of Technology in the Promotion of Children's Emotional Intelligence: The Multimedia Program" Developing Emotional Intelligence". International Journal of Emotional Education, 10(1), 47-67.

Davies, K. A., Lane, A. M., Devonport, T. J., y Scott, J. A. (2010). Validity and reliability of a brief emotional intelligence scale (BEIS-10). Journal of Individual Differences, 31, 198-208. DOI: https:// doi.org/10.1027/1614-0001/a000028

* Davis, S. K., Nowland, R., y Qualter, P. (2019). The role of emotional intelligence in the maintenance of depression symptoms and loneliness among children. Frontiers in psychology, 10, 1672. DOI: https:// doi.org/10.3389/fpsyg.2019.01672

* de las Heras Pérez, M. A., Indiano, R. R., de Orta Velo, Á., y Fernández, R. R. (2017). Diagnóstico de la autopercepción SocioEmocional de un grupo de alumnos de $5^{\circ}$ de Primaria tras la puesta en práctica de un Proyecto de Investigación Escolar para el estudio de los seres vivos desde el entorno próximo. Investigación en la Escuela, (91), 56-71. DOI: http://dx.doi. org/10.12795/IE.2017.i91.04

* dos Santos, N. D. N., y Franco, G. (2014). Inteligencia emocional y actividad lectora en una escuela de 1. ${ }^{\circ}$ ciclo. Revista INFAD de Psicología. International Journal of Developmental and Educational Psychology., 5(1), 159-166.

* Duncan, L. R., Bertoli, M. C., LatimerCheung, A. E., Rivers, S. E., Brackett, M. A., y Salovey, P. (2013). Mapping the protective pathway of emotional intelligence in youth: From social cognition to smoking intentions. Personality and individual differences, 54(4), 542544. DOI: https://doi.org/10.1016/i. paid.2012.09.002
Eaton, S. E. Coe, R., Waring, M., Hedges, LV, y Arthur, J. (Eds.). (2017). Research methods and methodologies in education. Thousand Oaks, CA: Sage.

Ekman, P., y Friesen, W. V. (1971). Constants across cultures in the face and emotion. Journal of personality and social psychology, 17(2), 124. DOI: https://doi.org/10.1037/ $\underline{\mathrm{h} 0030377}$

*El Hassan, K., y Kahil, R. (2005). The effect of "Living Values: An educational program" on behaviors and attitudes of elementary students in a prívate school in Lebanon. Early Childhood Education Journal, 33(2), 81-90. DOI: https://doi.org/10.1007/s10643-005$\underline{0028-0}$

* Esturgó-Deu, M. E., y Roca, J. S. (2019). The Relationship between Group Class Integration, Socio-emotional Abilities and Disruptive Behaviours Peer Group Integration Factors. Anuario de psicología/The UB Journal of psychology, 49(2), 85-93. DOI: https://doi. org/10.1344/anpsic2019.49.9

Extremera, N., Salguero, J. M., y FernándezBerrocal, P. (2011). Trait meta-mood and subjective happiness: A 7-week prospective study. Journal of Happiness Studies, 12(3), 509-517. DOI: https://doi.org/10.1007/ s10902-010-9233-7

Extremera Pacheco, N., Mérida-López, S., y Sánchez-Gómez, M. (2019). La importancia de la inteligencia emocional del profesorado en la misión educativa: impacto en el aula y recomendaciones de buenas prácticas para su entrenamiento.

Fernández-Berocal, P., Extremera N. y Ramos, N. (2004). Validity and reliability of the spanish modified version of the Trait Meta-Mood Scale. Psychological Reports, 94, 751-755. DOI: https://doi.org/10.2466/pr0.94.3.751-755

Ferragut, M., y Fierro, A. (2012). Emotional intelligence, well-being and academic achievement in preadolescents. Revista Latinoamericana de Psicología, 44(3), 95104.

* Ferrándiz, C., Hernández, D., Bermejo, R., Ferrando, M., y Sáinz, M. (2012). Social and emotional intelligence in childhood and adolescence: Spanish validation of a measurement instrument. Revista de Psicodidáctica, 17(2), 309-339. DOI: https:// doi.org/10.1387/Rev.Psicodidact.4496 
* Ferrando, M., Prieto, M. D., Almeida, L. S., Ferrándiz, C., Bermejo, R., LópezPina, J. A., ... y Fernández, M. C. (2011). Trait emotional intelligence and academic performance: Controlling for the effects of IQ, personality, and self-concept. Journal of Psychoeducational Assessment, 29(2), 150-159. DOI: https://doi. org/10.1177/0734282910374707

Furnham, A., y Petrides, K. V. (2003). Trait emotional intelligence and happiness. Social Behavior and Personality: an international journal, $37(8), 815$ 823. DOI: https://doi.org/10.2224/ sbp.2003.31.8.815

Gardner, H. (1983). Frames of mind: The theory of multiple intelligences. New York: Basic Books.

Goleman, D. (1995). Emotional intelligence. Bantam Books, Inc.

Goleman, D. (1996). Emotional intelligence. Why it can matter more than IQ. Learning, 24(6), 49-50.

Guil, R., Mestre, J.M., González, G. y Foncubierta, S. (2011). Integración del desarrollo de competencias emocionales en el currículum de Educación Infantil. Revista Electrónica Interuniversitaria de Formación del Profesorado, 14, 131-144. Recuperado de http://www. aufop.com/aufop/uploaded files/ articulos/1324678678.pdf

* Hansenne, M., y Legrand, J. (2012). Creativity, emotional intelligence, and school performance in children. International Journal of Educational Research, 53, 264-268. DOl: https://doi. org/10.1016/i.iier.2012.03.015

* Hassan, K. E., y Mouganie, Z. (2014). Implementation of the social decisionmaking skills curriculum on primary students (Grades 1-3) in Lebanon. School Psychology International, 35(2), 167-175. DOI: https://doi. org/10.1177/0143034312469758

* Hsieh, Y. P., Wei, H. S., Hwa, H. L., Shen, A. C. T., Feng, J. Y., y Huang, C. Y. (2019). The effects of peer victimization on children's Internet addiction and psychological distress: The moderating roles of emotional and social intelligence. Journal of Child and Family Studies, 28(9), 2487-2498.
DOI: $\quad$ https://doi.org/10.1007/s10826018-1120-6

* Huang, C. Y., Shen, A. C. T., Hsieh, Y. P., Feng, J. Y., Wei, H. S., Hwa, H. L., y Feng, J. Y. (2017). Cultural perspective on parenting, trait emotional intelligence and mental health in Taiwanese children.

* laosanurak, C., Chanchalor, S., y Murphy, E. (2016). Social and emotional learning around technology in a cross-cultural, elementary classroom. Education and Information Technologies, 21(6), 1639. 1662. DOI: https://doi.org/10.1007/ s10639-015-9406-4

* Jellesma, F. C., Rieffe, C., Meerum Terwogt, M., y Westenberg, P.M. (2011). Children's sense of coherence and trait emotional intelligence: $\mathrm{A}$ longitudinal study exploring the development of somatic complaints. Psychology and Health, 26(3), 307-320. DOI: https://doi. org/10.1080/08870440903411021

* Kim, H. S., y Kim, H. S. (2018). Effect of a musical instrument performance program on emotional intelligence, anxiety, and aggression in Korean elementary school children. Psychology of Music, 46(3), 440-453. DOl: https://doi. org/10.1177/0305735617729028

* Kokkinos, C. M., y Kipritsi, E. (2012). The relationship between bullying, victimization, trait emotional intelligence, self-efficacy and empathy among preadolescents. Social psychology of education, 15(1), 41-58. DOI: https://doi.org/10.1007/s $11218-011$ 9168-9

Lane, R. D., y Schwartz, G. E. (1987). Levels of emotional awareness: a cognitivedevelopmental theory and its application to psychopathology. The American journal of psychiatry.

* Li, D., Liu, T., Zhang, X., Wang, M., Wang, D., y Shi, J. (2017). Fluid intelligence, emotional intelligence, and the lowa Gambling Task in children. Intelligence, 62, 167-174. DOI: https://doi.org/10.1016/i. intell.2017.04.004

Ley Orgánica de Modificación de la Ley Orgánica de Educación (LOMLOE). Ley Orgánica 3/2020. 29 de diciembre de 2020. (España).

* Mancini, G. (2019). Trait emotional intelligence and draw-a-person emotional 
indicators: a first study on 8-year-old Italian children. Child Indicators Research, 12(5), 1629-1641. DOI: https://doi.org/10.1007/ s12187-018-9601-0

* Mateu-Martínez, Ornela, Piqueras, José Antonio, Jiménez-Albiar, $M^{a}$ Isabel, Espada, José Pedro, Carballo, José Luis, y Orgilés, Mireia. (2013). Eficacia de un programa de prevención cognitivo-conductual breve del rechazo social en niños. Terapia psicológica, $37(2)$. DOI: http://dx.doi.org/10.4067/ S0718-48082013000200005

* Mavroveli, S., Petrides, K. V., Shove, C., y Whitehead, A. (2008). Investigation of the construct of trait emotional intelligence in children. European child \& adolescent psychiatry, 17(8), 516-526. DOI: https:// doi.org/10.1007/s00787-008-0696-6

* Mavroveli, S., Petrides, K. V., Sangareau, Y., y Furnham,A. (2009). Exploring the relationships between trait emotional intelligence and objective socio emotional outcomes in childhood. British Journal of Educational Psychology, 79(2), 259-272. DOI: https:// doi.org/10.1348/000709908X368848

* Mavroveli, S., y Sánchez-Ruiz, M. J. (2011). Trait emotional intelligence influences on academic achievement and school behaviour. British Journal of Educational Psychology, $81(1), 112-134$. DOI: https:// doi.org/10.1348/2044-8279.002009

Mayer, J. D., y Salovey, P. (1997). What is emotional intelligence. Emotional development and emotional intelligence: Educational implications, 3, 31.

Mayer, J. D., Salovey, P., y Caruso, D. R. (2005). Mayer-Salovey-Caruso Emotional Intelligence Test: Youth Version (MSCEIT:YV): Item booklet. Toronto, Ontario, Canada: Multi-Health Systems.

Mayer, J. D., Caruso, D. R., y Salovey, P. (2016). Seven principles of emotional intelligence the ability model of emotional intelligence: principles and updates. Emotion Review, 8(4), 29. DOI: https://doi. org/10.1177/1754073916639667

Moon, Y. L. (1996). Measuring emotional intelligence: a case in Korea. SNU Journal of Education Research, 6.

Moon, Y. R. (1997). EQ Scale. Seúl, Corea del Sur: Instituto Daekyo para la Educación y la Ciencia. Daekyo SA.
O'Connor, PJ., Hill, A., Kaya, M., y Martin, B. (2019) The Measurement of Emotional Intelligence: A Critical Review of the Literature and Recommendations for Researchers and Practitioners. Front. Psychol. 10:1116. DOI: https://doi. org/10.3389/fpsyg.2019.01116

* Ordóñez, A., Maganto, C., y González, R. (2015). Somatic complaints, emotional awareness and maladjustment in schoolchildren. Anales de Pediatría (English Edition), 82(5), 308-315. DOI: $\quad$ https://doi.org/10.1016/i. anpede.2015.04.004

* Operto, F. F., Precenzano, F., Bitetti, I., Lanzara, V., Fontana, M. L., Pastorino, G. M. G., y Roccella, M. (2019). Emotional intelligence in children with severe sleeprelated breathing disorders. Behavioural neurology, 2019. DOI: https://doi. $\mathrm{org} / 10.1155 / 2019 / 6530539$

* Pandya, S. P. (2017). Spiritual education program for improving the emotional intelligence of gifted children: A multicity single-group evaluation study. Gifted and Talented International, 32(2), 120. 133. DOI: https://doi.org/10.1080/153 32276.2018 .1537138

* Parisi, L., Salerno, M., Maltese, A., Tripi, G., Romano, P., Di Folco, A., y Roccella, M. (2017). Emotional intelligence and obstructive sleep apnea syndrome in children: preliminary case-control study. Acta Medica Mediterranea, 33, 485. DOI: https://doi.org/10.19193/0393638420173072

* Peachey, A. A., Wenos, J., y Baller, S. (2017). Trait emotional intelligence related to bullying in elementary school children and to victimization in boys. OTJR: occupation, participation and health, 37(4), 178-187. DOI: https:// doi.org/10.1177/1539449217715859

Pena, M. y Repetto, E. (2008). Estado de la investigación en España sobre Inteligencia Emocional en el ámbito educativo. Revista Electrónica de Investigación Psicoeducativa, 6(15), 400-420.

* Pérez-Escoda, N., Filella, G., Alegre, A., y Bisquerra, R. (2012). Developing the emotional competence of teachers and pupils in school contexts. 
Petrides, K. V., y Furnham, A. (2000). On the dimensional structure of emotional intelligence. Personality and individual differences, 29(2), 313-320. DOI: https://doi.org/10.1016/S01918869(99)00195-6

* Petrides, K. V., Sangareau, Y., Furnham, A., y Frederickson, N. (2006). Trait emotional intelligence and children's peer relations at school. Social Development, 15(3), 537-547. DOI: https://doi.org/10.1111/ j. 1467-9507.2006.00355.x

Pons, F., Harris, P., y de Rosnay, M. (2004). Emotion comprehension between 3 and 11 years: Developmental periods and hierarchical organization. European journal of developmental psychology $7(2), \quad 127-152 . \quad$ DOI: $\quad$ https://doi. org/10.1080/17405620344000022

Prieto, M. D., Ferrándiz, C., Ferrando, M., Sánchez, C., y Bermejo, R. (2008). Inteligencia emocional y alta habilidad. Revista española de pedagogía, 241-259.

Pulido, F., y Herrera, F. (2016). La inteligencia emocional como predictora del rendimiento académico infantil: el contexto pluricultural de Ceuta. Revista Complutense de Educación, 28, 12511265. https://revistas. ucm.es/index.php/ RCED/article/view/51712/51786. DOI: https://doi.org/10.5209/RCED.51712

* Pulido-Acosta, F., y Herrera-Clavero, F. (2019). Predicting children's academic achievement through emotional intelligence. PSICOLOGIA EDUCATIVA, 25(1), 23-30. DOI: https://doi. org/10.5093/psed2018a16

* Qualter, P., Barlow, A., y Stylianou, M. S. (2011). Investigating the relationship between trait and ability emotional intelligence and theory of mind. British Journal of Developmental Psychology, 29(3), 437-454. DOl: https://doi. org/10.1348/026151010X502999

* Qualter, P., Urquijo, I., Henzi, P., Barrett, L., y Humphrey, N. (2019). Ability emotional intelligence and children's behaviour in the playground. Social Development, 28(2), 430-448. DOI: https://doi.org/10.1111/ sode. 12340

Rieffe, C., Meerum Terwogt, M., Petrides, K. V., Cowan, C., Miers, A. C., y Tolland,
A. (2007). Psychometric properties of the Emotion Awareness Questionnaire for children. Personality and Individual Differences, 43, 95-105. DOI: https://doi. org/10.1016/i.paid.2006.11.015

Rieffe, C., Oosterveld, P., Miers, A. C., Meerum Terwogt, M., y Ly, V. (2008). Emotion awareness and internalising symptoms in children and adolescents: The Emotion Awareness Questionnaire revised. Personality and Individual Differences, 45, 756-761. DOI: https://doi.org/10.1016/i. paid.2008.08.001

Rockhill, C. M., y Greener, S. H. (15-18 de abril de 1999). Development of the Trait Meta-Mood Scale for Elementary School Children. Poster presentado en la Reunión Bienal de la Sociedad de Investigación en Desarrollo Infantil. Albuquerque, Nuevo México. Estados Unidos. https://files.eric. ed.gov/fulltext/ED430688.pdf

* Ros Morente, A., Filella Guiu, G., Ribes, R., y Pérez Escoda, N. (2017). Análisis de la relación entre competencias emocionales, autoestima, clima de aula, rendimiento académico y nivel de bienestar en una muestra de escolares de educación primaria. Revista Española de Orientación y Psicopedagogía, 2017, vol. 28, núm. 1, p. 8-18.

* Russo, P. M., Mancini, G., Trombini, E., Baldaro, B., Mavroveli, S., y Petrides, K. V. (2012). Trait emotional intelligence and the Big Five: A study on Italian children and preadolescents. JournalofPsychoeducational Assessment, 30(3), 274-283. DOI: https:// doi.org/10.1177/0734282911426412

Salguero, J. M., Fernández-Berrocal, P., RuizAranda, D., Castillo, R., y Palomera, R. (2011). Inteligencia emocional y ajuste psicosocial en la adolescencia: El papel de la percepción emocional. European journal of education and psychology, 4(2), 143152. DOI: https://doi.org/10.1989/ejep. $\mathrm{v} 4 \mathrm{i} 2.84$

Salovey, P., y Mayer, J. D. (1989-1990). Emotional intelligence. Imagination, Cognition and Personality, 9(3), 185-211. DOI: https://doi.org/10.2190/DUGGP24E-52WK-6CDG

Salovey, P., Mayer, J. D., Goldman, S. L., Turvey, C; y Palfai, T. P. (1995). Emotional 
attention, ciarity, and repair: exploring emotional intelligence using the Trait Meta-Mood Scale. En J. W. Pennebaker (Ed.), Emotion, Disclosure, \& Health (pp. 125- 154). Washington: American Psychological Association. DOI: https:// doi.org/10.1037/10182-006

* Sánchez, M. C. B., y Molero, D. (2014). Estudio sobre inteligencia emocional y afectos en escolares de educación primaria. Revista Electrónica de Investigación y Docencia (REID), (12). DOl: https://doi. org/10.17561/reid

Sánchez-Teruel, D. (2009). Actualización en inteligencia emocional. Madrid. Editorial CEP Sánchez-Teruel, D.(2009). Updated on emotional intelligence. Madrid. Editorial CEP.

Sánchez-Teruel, D., y Robles-Bello, M. A. (2018). Instrumentos de evaluación en inteligencia emocional: una revisión sistemática cuantitativa. Perspectiva Educacional, 57(2), 27-50. DOI: https:// doi.org/10.4151/07189729-Vol.57-

Iss.2-Art.712

* Sastre, S., Artola, T., y Alvarado, J. M. (2019). Emotional intelligence in elementary school children. EMOCINE, a novel assessment test based on the interpretation of cinema scenes. Frontiers in psychology, 10, 1882. DOI: https://doi.org/10.3389/ fpsyg.2019.01882

* Stassart, C., Dardenne, B., y Etienne, A. M. (2017b). The role of parental anxiety sensitivity and learning experiences in children's anxiety sensitivity. British Journal of Developmental Psychology, 35(3), 359375. DOI: https://doi.org/10.1111/ bidp. 12172

* Stassart, C., Etienne, A. M., Luminet, O., Kaïdi, I., y Lahaye, M. (2017a). The psychometric properties of the French version of the trait emotional intelligence questionnaire-child short form. Journal of Psychoeducational Assessment, 37(3), 293-306. DOI: https:// doi.org/10.1177/0734282917740499

Sucaromana, U. (2010). The Thai emotional intelligence screening test: measurement and validation with pre-service teachers. Unpublished doctoral dissertation, Griffith University, Logan. DOI: https://doi. org/10.25904/1912/1500
Sullivan, A. K. (1999). The Emotional Intelligence Scale for Children. Unpublished doctoral dissertation. University of Virginia, Charlottesville, Virginia, United States. Retrieved April 25, 2013, from Umi Proquest database. DOI: https://doi.org/10.18130/V38C43

Schutte, N. S., Malouff, J. M., Hall, L. E., Haggerty, D. J., Cooper, J. T., y Golden, C. J. (1998). Development and validation of a measure of emotional intelligence. Personality and Individual Differences. DOI: $\quad$ https://doi.org/10.1016/S01918869(98)00001-4

Stylianou, M.S. (2007). Does Executive Functioning Training Improve Mentalising Ability? Ph.D. thesis, University of Liverpool. Takšić, V. (2002). The importance of emotional intelligence (competence) in positive psychology. Trabajo presentado en 1st International Positive Psychology Summit, Washington, DC.

Takšić, V., Mohorić, T., y Duran, M. (2009). Emotional skills and competence questionnaire (ESCQ) as a self-report measure of emotional intelligence. Horizons of Psychology, 18(3), 7-21.

* Tannous, A., y Matar, J. (2010). The relationship between depression and emotional intelligence among a sample of Jordanian children. Procedia-Social and Behavioral Sciences, 5, 1017 1022. DOI: https://doi.org/10.1016/i. sbspro.2010.07.228

Thorndike, E. L. (1920). Intelligence and its use. Harper's Magazine, 140, 227-235.

Titrek, O. (2005). Duygusal Zeka Yeterliklerini İş Yaşamında Kullanma Düzeyi Ölçeğitnin Geliştirilmesi: Geçerlik ve Güvenirlik Çalışmaları. Türk Psikolojik Danışma ve Rehberlik Dergisi, 3(24), 73-87.

* Turanzas, J. A., Cordón, J. R., Choca, J. P., y Mestre, J. M. (2020). Evaluating the APAC (mindfulness for giftedness) program in a Spanish sample of gifted children: a pilot study. Mindfulness, $17(1)$, 86-98. DOl: https://doi.org/10.1007/s 12671-0180985-1

* Van Dyke, C. J., y Elias, M. J. (2008). How Expressions of Forgiveness, Purpose, and Religiosity Relate to Emotional Intelligence and Self-Concept in Urban 
Fifth-Grade Students. American Journal of Orthopsychiatry, 78(4), 48 1-493. DOI: https://doi.org/10.1037/a0014451

* Williams, C., Daley, D., Burnside, E., y Hammond-Rowley, S. (2009). Measuring emotional intelligence in preadolescence. Personality and Individual Differences, 47(4), 316-320. DOI: https://doi. org/10.1016/i.paid.2009.03.019

* Williams, C., Daley, D., Burnside, E., y Hammond-Rowley, S. (2010a). Does item overlap account for the relationship between trait emotional intelligence and psychopathology in preadolescents? Personality and Individual Differences, 48(8), 867-871. DOI: https://doi. org/10.1016/i.paid.2010.02.006

* Williams, C., Daley, D., Burnside, E., y Hammond-Rowley, S. (2010b). Can trait Emotional Intelligence and objective measures of emotional ability predict psychopathology across the transition to secondary school? Individual Differences, 48(2), 161 165. DOI: https://doi.org/10.1016/i. paid.2009.09.014

* Windingstad, S., McCallum, R. S., Mee Bell, S., y Dunn, P. (2011). Measures of emotional intelligence and social acceptability in children: A concurrent validity study. Canadian Journal of School Psychology, 26(2), 107-126. DOI: https:// doi.org/10.1177/0829573511406510

* Yang, J. C., Quadir, B., y Chen, N. S. (2019). Effects of Children's Trait Emotional Intelligence on Digital GameBased Learning. International Journal of Human-Computer Interaction, 35(4-5), 374-383. DOI: https://doi.org/10.1080 $\angle 10447318.2018 .1543088$ 